\title{
Specialization of Formal Cohomology and Asymptotic Expansions
}

By

\author{
Violaine CoLIN*
}

\begin{abstract}
Following a work of Andronikof, we build a theory of Whitney specialization for $C^{\infty}$-functions and holomorphic functions. To a $\mathbb{R}$-constructible sheaf, we associate a $\mathcal{D}$-module. In particular, the constant sheaf gives the germs of functions asymptotically developable.
\end{abstract}

\section{Contents}

Introduction

$\S 1 . \quad$ Notations

\$1.1. Whitney functions

$\S 1.2$. Whitney functor

$\S 1.3$. Normal deformation

$\S 2$. Whitney Specialization

$\S 2.1$. Specialization of the Whitney functor

\$2.2. Asymptotic expansions for $\boldsymbol{C}^{\infty}$-functions

$\S 2.3$. Operations

$\S 3 . \quad$ Formal Specialization

$\S 3.1$. Specialization of the functor of formal cohomology

§3.2. Complex normal deformation

$\S 3.3 . \quad$ Examples

§3.3.1. Complexification of a real manifold

§3.3.2. Asymptotic expansions for holomorphic functions

$\S 3.4$. Operations

Communicated by M. Kashiwara, April 4, 2000.

2000 Mathematics Subject Classification(s): 32C38, 34E05.

Key words: $\mathcal{D}$-modules, specialization, asymptotic expansions.

Supported by JSPS P 97212 and TMR ERBFMRXCT960040.

*CMAF, Univ. de Lisboa, Avenida Professor Gama Pinto, 2, 1649-003 Lisboa, Portugal. 


\section{Introduction}

The idea of "algebraic analysis", according to Mikio Sato, is to consider the classical analysis with algebraic tools such as sheaf theory, homological algebra.

In this paper, we consider the notion of asymptotic expansions in the frame work of $\mathcal{D}$-modules. The idea is that asymptotically developable functions on sector are related to Whitney functions on closed subsectors.

A first step was then to translate the Whitney functions in terms of $\mathcal{D}$ modules. However, sheaves can express only local properties, and some difficulties appear when we want to consider the growth conditions. In 1983, Kashiwara overcame these difficulties for distributions. Constructing an inverse functor of $R \mathcal{H o m}\left(\cdot, \mathcal{O}_{X}\right)$ in the Riemann-Hilbert correspondence [K], he obtained two functors, the Schwartz functor and the functor of moderate cohomology, its Dolbeault complex. Roughly speaking, the Schwartz functor associates to a subanalytic open subset $U$ the moderate distributions on $U$, and to a subanalytic closed subset $Z$ the distributions supported by $Z$.

Finding a functorial expression of growth conditions for distributions, it then seems natural to look at its dual. In 1994, Kashiwara and Schapira gave a new construction for the preceding functors [KS1]. The method used allowed them to define their "dual", the Whitney functor and its Dolbeault complex, the functor of formal cohomology. Roughly speaking, the Whitney functor associates to $U$ the $C^{\infty}$ functions vanishing on the complementary of $U$ up to the infinite order, and to $Z$ the infinitely differentiable functions on $Z$ in the sense of Whitney.

The second step is to look those Whitney functions in sectors, in other words to specialize the Whitney functor. That was a suggestion of Schapira for my thesis. So following the path drawn by Andronikof for the functor of moderate cohomology [A], we adapted the specialization to the functor of formal cohomology [C1].

In the first two chapters, $X$ designates a real analytic manifold. In chapter 1 , we recall the definition of infinitely differentiable functions in the sense of Whitney, the construction of Whitney functor and some notations used in the specialization of a sheaf along a submanifold $M$ of $X$.

In chapter 2, we adapt the specialization to the Whitney functor. We obtain a functor of Whitney specialization along $M$, which to a $\mathbb{R}$-constructible sheaf on $X$ associates a conic sheaf on $T_{M} X$. In particular, its value on the constant sheaf on $X$ is the sheaf $w \nu_{M}\left(\mathcal{C}_{X}^{\infty}\right)$ of germs of $C^{\infty}$ functions in a normal cone to $M$ in $X$, all derivatives of which are asymptotically developable along $M$, and its value on the sheaf $\mathbb{C}_{X \backslash M}$ is the subsheaf $w^{0} \nu_{M}\left(\mathcal{C}_{X}^{\infty}\right)$ of functions all 
derivatives of which have zero as asymptotic expansion.

In the third chapter, $X$ is a complex analytic manifold and $M$ is a real analytic submanifold of the underlying real manifold of $X$. Then the specialization along $M$ of the formal cohomology is the Dolbeault complex of the Whitney specialization. In particular if $M$ is a smooth complex submanifold of $X$, the image $w \nu_{M}\left(\mathcal{O}_{X}\right)$ of the constant sheaf on $X$ is the sheaf of germs of holomorphic functions in sectors of $X$ asymptotically developable along $M$, having asymptotic expansion with holomorphic coefficients on $M$. In the case where $M$ is the origin in $\mathbb{C}$, we recover Malgrange-Sibuya's construction. If $X$ is a complexification of $M, w \nu_{M}\left(\mathcal{O}_{X}\right)$ is a subsheaf of the inverse image of $\mathcal{C}_{M}^{\infty}$ in $T_{M} X$, describing the infinitely differentiable functions on $M$ which are boundary value along $M$ of a holomorphic function in a sector.

To study the solutions of differential systems, it seems more efficient to look at the microlocalization. For instance, in comparison theorem, the noncharacteristic property appears naturally in the cotangent bundle. So the next step is to transpose this present construction to the frame of microlocal analysis [SKK]. In [C2], we gave a construction equivalent to the functors $\mu h o m\left(\cdot, \mathcal{O}_{X}\right)$ and $\mathcal{T} \mu h o m\left(\cdot, \mathcal{O}_{X}\right)$ for the formal cohomology and obtain an action of microdifferential operators. The big machinery being installed, we can expect it to be as fruitful as it have been in moderate case.

I thank Pierre Schapira for the suggestion of this construction and to have supervised my thesis. I am particularly grateful to Masaki Kashiwara for the invitations at RIMS during my thesis and for a post-doctoral position, for all the discussions and proof-relectures. I would like also to thank Teresa Monteiro Fernandes for the invitation at CMAF/UL and the final push.

\section{$\S 1$. Notations}

Let $X$ be a real analytic manifold. We denote by $\mathcal{A}_{X}, \mathcal{C}_{X}^{\infty}$ and $\mathcal{D}_{X}$ the sheaves on $X$ of complex valued real analytic functions, $C^{\infty}$-functions and finite-order differential operators with coefficients in $\mathcal{A}_{X}$, respectively.

\section{\$1.1. Whitney functions}

For more details, we refer to the book of Malgrange [Ml2], and the papers of Whitney [Wh1][Wh2]. In this paragraph, we set $X=\mathbb{R}^{n}$. Let $A$ be a subset of $X$.

Definition 1.1. One calls infinitely differentiable function on $A$ in the sense of Whitney or Whitney function on A a family $F=\left(F^{k}\right)_{k \in \mathbb{N}^{n}}$ of continuous functions on A such that: 
$\forall m \in \mathbb{N}, \quad \forall k \in \mathbb{N}^{n},|k| \leqslant m, \quad \forall a \in A, \quad \forall \varepsilon>0$, there exists a neighborhood $U$ of a in $X$ such that $\forall x, y \in U \cap A$

$$
\left|F^{k}(x)-\sum_{|j| \leqslant m} \frac{(x-y)^{j}}{j !} F^{j+k}(y)\right| \leqslant \varepsilon d(x, y)^{m} .
$$

We shall denote by $W_{X, A}^{\infty}$ the space of Whitney functions on $A$.

Let $U$ be an open subset of $X$. If $F$ is a Whitney function on $U$ then we notice that $F^{0} \in \mathcal{C}_{X}^{\infty}(U)$ and $D^{k} F^{0}=F^{k}$. Conversely if $f \in \mathcal{C}_{X}^{\infty}(U)$, Taylor's expansion shows that the family $\left(D^{k} f\right)_{k \in \mathbb{N} n}$ is a Whitney function on $U$.

Theorem 1.2 (Whitney [Wh1]). Let $Z$ be a closed subset of $X, U$ an open neighborhood of $Z$ and $F$ a Whitney function on $Z$. Then there exists a function $f$ in $\mathcal{C}_{X}^{\infty}(U)$ such that $\left.D^{k} f\right|_{Z}=F^{k}$.

This is a generalization of the Borel theorem ( $Z$ is a point): for any formal series, there exists a $C^{\infty}$ function on $X$ such that the $n$-th derivative at this point is equal to the $n$-th coefficient of the series for all integer $n$. In fact, we have the exact sequence of Fréchet spaces:

$$
0 \rightarrow \mathcal{I}_{X, Z}^{\infty}(U) \rightarrow \mathcal{C}_{X}^{\infty}(U) \rightarrow W_{X, Z}^{\infty} \rightarrow 0
$$

where $\mathcal{I}_{X, Z}^{\infty}$ is the subsheaf of $\mathcal{C}_{X}^{\infty}$ consisting of functions vanishing on $Z$ up to the infinite order. An important well-known remark is that: $W_{X, Z}^{\infty} \neq$ $\Gamma\left(Z ; \mathcal{C}_{X}^{\infty}\right) \simeq \underset{\lim _{U \supset Z}}{\mathcal{C}_{X}^{\infty}}(U)$. Nevertheless the presheaf $U \mapsto W_{U, U \cap Z}^{\infty}$, is a sheaf that we shall denote by $\mathcal{W}_{X, Z}^{\infty}$. We get the exact sequence of sheaves:

$$
0 \rightarrow \mathcal{I}_{X, Z}^{\infty} \rightarrow \mathcal{C}_{X}^{\infty} \rightarrow \mathcal{W}_{X, Z}^{\infty} \rightarrow 0
$$

A subset $A$ of $X$ is said regular if for all $x$ in $X$, there exists a neighborhood $U$ of $x$ and a positive constant $C$ such that for all $y$ and $z$ in $A \cap U$, there exists a rectificable curve in $A \cap U$ from $y$ to $z$ whose length is smaller than $C d(y, z)$. Obviously, any convex subset is regular. Let us also recall that any closed subanalytic subset is regular. On the other hand an open subanalytic subset is not necessarily regular (for instance the complementary of a closed half line).

Theorem 1.3 (Whitney [Wh2]). Let $U$ be a regular open subset and $f \in \mathcal{C}_{X}^{\infty}(U)$ such that for all $k \in \mathbb{N}^{n}, D^{k} f$ admits an extension to a continuous function on $\bar{U}$. Then there exist a Whitney function on $\bar{U}, F$ such that $F^{k} \mid U=$ $D^{k} f$. 


\section{$\S 1.2$. Whitney functor}

We shall recall briefly the construction of the Whitney functor. For more details, see [KS1].

The Whitney functor, denoted by $\cdot \stackrel{\mathrm{w}}{\otimes} \mathcal{C}_{X}^{\infty}$ is an exact functor from the category of $\mathbb{R}$-constructible sheaves to the category of $\mathcal{D}_{X}$-modules. Its value on $\mathbb{C}_{U}$ for an open subanalytic subset $U$ of $X$ is the subsheaf $\mathcal{I}_{X, X \backslash U}^{\infty}$ of $\mathcal{C}_{X}^{\infty}$ consisting of sections vanishing at infinite order on $X \backslash U$.

Notice that $\mathbb{C}_{Z} \stackrel{\mathrm{w}}{\otimes} \mathcal{C}_{X}^{\infty}$ is nothing but the sheaf $\mathcal{W}_{X, Z}^{\infty}$ of Whitney functions on $Z$.

This functor being exact, it extends naturally to a functor from the derived category $\mathbf{D}_{\mathbb{R}-\mathrm{c}}^{\mathrm{b}}\left(\mathbb{C}_{X}\right)$ of $\mathbb{C}_{X}$-modules with bounded and $\mathbb{R}$-constructible cohomology to the derived category $\mathbf{D}^{\mathrm{b}}\left(\mathcal{D}_{X}\right)$ of $\mathcal{D}_{X}$-modules with bounded cohomology.

Recall the operations on the Whitney functor. Let $f: Y \rightarrow X$ be a morphism of real analytic manifolds, $F$ and $G$ objects of $\mathbf{D}_{\mathbb{R}-\mathrm{c}}^{\mathrm{b}}\left(\mathbb{C}_{X}\right)$ and $\mathbf{D}_{\mathbb{R}-\mathrm{c}}^{\mathrm{b}}\left(\mathbb{C}_{Y}\right)$, respectively. We denote by $\mathcal{D}_{Y \rightarrow X}=\mathcal{A}_{Y} \underset{f^{-1} \mathcal{A}_{X}}{\otimes} f^{-1} \mathcal{D}_{X}$, the transfer bi-module for $\mathcal{D}$-modules.

In $\mathbf{D}^{\mathrm{b}}\left(\mathcal{D}_{X}\right)$, we have the morphism:

$$
R f_{!} G \stackrel{\mathrm{w}}{\otimes} \mathcal{C}_{X}^{\infty} \rightarrow R f_{*}\left(R \mathcal{H}{ }^{\infty} m_{\mathcal{D}_{Y}}\left(\mathcal{D}_{Y \rightarrow X}, G \stackrel{\mathrm{w}}{\otimes} \mathcal{C}_{Y}^{\infty}\right)\right),
$$

which is an isomorphism if $f$ is proper on $\operatorname{supp}(G)$.

In $\mathbf{D}^{\mathrm{b}}\left(\mathcal{D}_{Y}\right)$, we have the morphism:

$$
\mathcal{D}_{Y \rightarrow X} \underset{f^{-1} \mathcal{D}_{X}}{\stackrel{L}{\otimes}} f^{-1}\left(F \stackrel{\mathrm{w}}{\otimes} \mathcal{C}_{X}^{\infty}\right) \rightarrow\left(f^{-1} F\right) \stackrel{\mathrm{w}}{\otimes} \mathcal{C}_{Y}^{\infty},
$$

which is an isomorphism if $f$ is a closed embedding.

In $\mathbf{D}^{\mathrm{b}}\left(f^{-1} \mathcal{D}_{X}\right)$, we have the morphism:

$$
f^{-1}\left(F \stackrel{\mathrm{w}}{\otimes} \mathcal{C}_{X}^{\infty}\right) \rightarrow R_{\mathcal{H}} \operatorname{Hom}_{\mathcal{D}_{Y}}\left(\mathcal{D}_{Y \rightarrow X},\left(f^{-1} F\right) \stackrel{\mathrm{w}}{\otimes} \mathcal{C}_{Y}^{\infty}\right),
$$

which is an isomorphism if $f$ is smooth.

In $\mathbf{D}^{\mathrm{b}}\left(\mathcal{D}_{X \times Y}\right)$, we have the natural morphism:

$$
\left(F \stackrel{\mathrm{w}}{\otimes} \mathcal{C}_{X}^{\infty}\right) \underline{\otimes}\left(G \stackrel{\mathrm{w}}{\otimes} \mathcal{C}_{Y}^{\infty}\right) \rightarrow(F \otimes G) \stackrel{\mathrm{w}}{\otimes} \mathcal{C}_{X \times Y}^{\infty} .
$$

Remark 1.4. Let $F \in \mathrm{Ob}\left(\mathbf{D}_{\mathbb{R}-\mathrm{b}}^{\mathrm{b}}\left(\mathbb{C}_{X}\right)\right)$. Let $U$ and $V$ be two subanalytic open subsets such that $\bar{V} \subset U$. Following Andronikof's factorization of the restriction morphisms for the functor $\mathcal{T} \operatorname{hom}\left(\cdot, \mathcal{D} b_{X}\right)$, we have the commutative 
diagram:

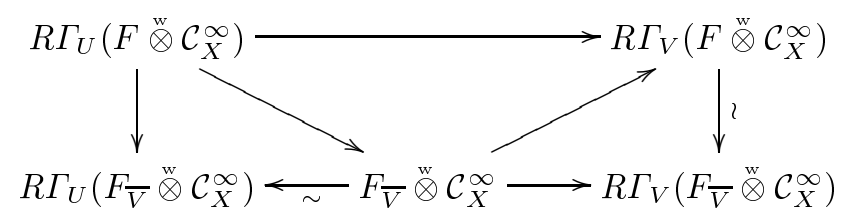

In particular, if $x$ is a point in $X$, we have the stalk's formula:

$$
H^{k}\left(F \stackrel{\mathrm{w}}{\otimes} \mathcal{C}_{X}^{\infty}\right)_{x} \simeq \underset{U}{\lim } \mathrm{R}^{k} \Gamma\left(X ; F_{\bar{U}} \stackrel{\mathrm{w}}{\otimes} \mathcal{C}_{X}^{\infty}\right),
$$

where $U$ ranges through a system of relatively compact subanalytic open neighborhoods of $x$ in $X$.

\section{$\S 1.3 . \quad$ Normal deformation}

Let us recall the construction given in $[\mathrm{KS} 2]$. Let $X$ be a real analytic manifold, $M$ a submanifold of $X$ and $T_{M} X \stackrel{\tau}{\rightarrow} M$ the normal bundle to $M$ in $X$. We denote by $k$ the embedding of $M$ as the zero-section in $T_{M} X$. We set $\dot{T}_{M} X=T_{M} X \backslash M$, and denote the projection on the sphere bundle by $\dot{\gamma}: \dot{T}_{M} X \rightarrow S_{M} X=\dot{T}_{M} X / \mathbb{R}_{+}^{*}$. We call normal deformation of $M$ in $X$, the data of:

i) a real analytic manifold $\tilde{X}$,

ii) a map $p: \tilde{X} \rightarrow X$ such that $p^{-1}(X \backslash M) \simeq(X \backslash M) \times \mathbb{R}^{*}$ and $p^{-1}(M) \simeq$ $T_{M} X \cup(M \times \mathbb{R})$ identifying $M \times\{0\}$ to the zero-section of $T_{M} X$,

iii) a map $t: \tilde{X} \rightarrow \mathbb{R}$ such that $t^{-1}\left(\mathbb{R}^{*}\right) \simeq X \times \mathbb{R}^{*}$ and $t^{-1}(0) \simeq T_{M} X$,

iv) a group action of $\mathbb{R}^{*}$ on $\tilde{X}$ such that the stalk of $p$ is stable by this action.

Let us denote by $\Omega$ the open subset $t^{-1}\left(\mathbb{R}_{+}^{*}\right)$. We have the following commutative diagram:

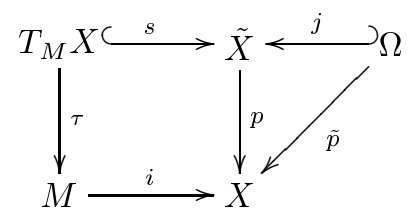

We denote by $\theta$ the infinitesimal generator of the action of $\mathbb{R}^{*}$ on $\tilde{X}$. At a point $\tilde{x}$ in $\tilde{X}, \theta_{\tilde{x}}=\left.\frac{d}{d c}(c . \tilde{x})\right|_{c=1}$. Let $x=\left(x^{\prime}, x^{\prime \prime}\right)$ be a local coordinate system on an 
open subset $U$ of $X$ such that $M$ is defined on $U$ by $\left\{x^{\prime}=0\right\}$. On $p^{-1}(U)$, we consider the coordinate system $\left(\tilde{x}^{\prime}, x^{\prime \prime}, t\right)$. We denote by $D_{x_{i}}=\frac{\partial}{\partial x_{i}}$. Then $p\left(\tilde{x}^{\prime}, x^{\prime \prime}, t\right)=\left(t \tilde{x}^{\prime}, x^{\prime \prime}\right)$

c. $\left(\tilde{x}^{\prime}, x^{\prime \prime}, t\right)=\left(c \tilde{x}^{\prime}, x^{\prime \prime}, c^{-1} t\right)$ $\theta=\left\langle\tilde{x}^{\prime}, D_{\tilde{x}^{\prime}}\right\rangle-t D_{t}$.

Let $A$ be a subset of $X$. The set $C_{M}(A)=T_{M} X \cap \overline{\tilde{p}^{-1}(A)}$ is called the normal cone of $A$ along $M$. Let $F \in \mathrm{Ob}\left(\mathbf{D}^{\mathrm{b}}(X)\right)$. The specialization of $F$ along $M$ is defined by:

$$
\nu_{M}(F)=s^{-1} R j_{*} \tilde{p}^{-1} F \simeq s^{!} R j_{!} \tilde{p}^{!} F .
$$

For $v \in T_{M} X$, let $\mathcal{U}$ be the family of subanalytic open subsets $U$ of $X$ such that $v$ is not in the normal cone $C_{M}(X \backslash U)$. Then we have the stalk's formula:

$$
H^{k}\left(\nu_{M}(F)\right)_{v} \simeq \underset{\lim _{U \in \mathcal{U}}}{ } \mathrm{R}^{k} \Gamma(U ; F) .
$$

Identifying $T_{M} M$ with $M$, we have

$$
\begin{aligned}
\left.\nu_{M}(F)\right|_{T_{M} M} & \left.\simeq F\right|_{M}, \\
\left.R \Gamma_{T_{M} M}\left(\nu_{M}(F)\right)\right|_{T_{M} M} & \left.\simeq R \Gamma_{M}(F)\right|_{M} .
\end{aligned}
$$

Recall that if $G$ is a conic object of $\mathbf{D}^{\mathrm{b}}\left(T_{M} X\right)$ then we have

$$
\left.R \tau_{*} G \simeq G\right|_{T_{M} M} \text { and }\left.R \tau_{!} G \simeq R \Gamma_{T_{M} M}(G)\right|_{T_{M} M} .
$$

\section{§2. Whitney Specialization}

In this chapter, $X$ will be a real analytic manifold, $M$ a real analytic submanifold of $X$ and $\tilde{X}$ the normal deformation of $X$ along $M$.

\section{$\S 2.1$. Specialization of the Whitney functor}

Let us first notice that if we just compose the functor of specialization of a sheaf along $M$ with the Whitney functor, we loose the growth conditions along $M$. For instance, we obtain the same object with the constant sheaf on $X$ and the sheaf $\mathbb{C}_{X \backslash M}$. In fact, for $v \in T_{M} X$, let $\mathcal{U}$ be the family of open subsets $U$ of $X$ such that $v \notin C_{M}(X \backslash U)$. We have the isomorphisms:

$$
\left(\nu_{M}\left(\mathbb{C}_{X} \stackrel{\mathrm{w}}{\otimes} \mathcal{C}_{X}^{\infty}\right)\right)_{v} \simeq \underset{\lim _{U \in \mathcal{U}}}{\mathcal{C}_{X}^{\infty}}(U) \simeq \underset{\lim _{U \in \mathcal{U}}}{\mathcal{I}_{X, M}^{\infty}}(U) \simeq\left(\nu_{M}\left(\mathbb{C}_{X \backslash M} \stackrel{\mathrm{w}}{\otimes} \mathcal{C}_{X}^{\infty}\right)\right)_{v} .
$$

Our aim is to construct a functor which will keep growth conditions on $M$ such as being infinitely differentiable on $M$ in the sense of Whitney or vanishing 
at infinite order on $M$. In fact what should appear is the inductive limits of $\Gamma\left(X ; \mathbb{C}_{\bar{U}} \stackrel{\mathrm{w}}{\otimes} \mathcal{C}_{X}^{\infty}\right)$ and $\Gamma\left(X ; \mathbb{C}_{\bar{U} \backslash M} \stackrel{\mathrm{w}}{\otimes} \mathcal{C}_{X}^{\infty}\right)$.

The first idea is to factorize the restriction morphisms $\mathcal{C}_{X}^{\infty}(U) \rightarrow \mathcal{C}_{X}^{\infty}\left(U^{\prime}\right)$

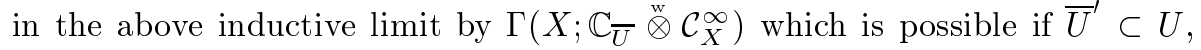
as in the commutative diagram (1.4). The problem is that for $U$ in $\mathcal{U}$, we can not always find an open set $U^{\prime}$ in $\mathcal{U}$ such that $\bar{U}^{\prime} \subset U$, if $v$ is outside the zero-section. But if we look in the normal deformation $\tilde{X}$, we can consider a base of neighborhoods $V$ of $v$ in $\tilde{X}$ whose projection on $X$ is $\mathcal{U}$. So we should apply the Whitney functor on $\tilde{X}$.

The second idea is that if we intersect $\bar{V}$ with $\Omega$ like in usual specialization

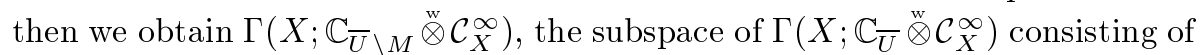
functions flat on $M$. As $\bar{U} \cap M$ corresponds to $\bar{V} \cap T_{M} X=\bar{V} \cap \partial \Omega$, we should intersect with $\bar{\Omega}$.

Finally we set the following definitions:

Definition 2.1. Let $F \in \operatorname{Ob}\left(\mathbf{D}_{\mathbb{R}-c}^{\mathrm{b}}\left(\mathbb{C}_{X}\right)\right)$. We set:

$$
w \nu_{M}\left(F, \mathcal{C}_{X}^{\infty}\right)=s^{-1} R \mathcal{H} o_{\mathcal{D}_{\tilde{X}}}\left(\mathcal{D}_{\tilde{X} \rightarrow X},\left(p^{-1} F\right)_{\bar{\Omega}} \stackrel{\mathrm{w}}{\otimes} \mathcal{C}_{\tilde{X}}^{\infty}\right) .
$$

We call $w \nu_{M}\left(F, \mathcal{C}_{X}^{\infty}\right)$ the Whitney specialization of $F$ along $M$.

We have the diagram:

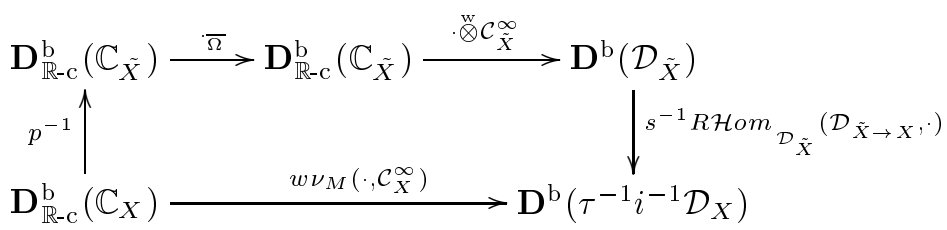

Proposition 2.2. Let $F \in \operatorname{Ob}\left(\mathbf{D}_{\mathbb{R}-c}^{\mathrm{b}}\left(\mathbb{C}_{X}\right)\right)$. In $\mathbf{D}^{\mathrm{b}}\left(\tau^{-1} \mathcal{D}_{X}\right)$, we have the commutative diagram:

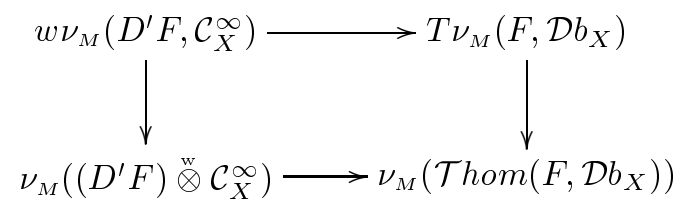

Proof. The first vertical arrow comes from the sequence of morphisms:

$$
\begin{aligned}
w \nu_{M}\left(D^{\prime} F, \mathcal{C}_{X}^{\infty}\right) & =s^{-1} R \mathcal{H o m}_{\mathcal{D}_{\tilde{X}}}\left(\mathcal{D}_{\tilde{X} \rightarrow X},\left(p^{-1}\left(D^{\prime} F\right)\right)_{\bar{\Omega}} \stackrel{\mathrm{w}}{\otimes} \mathcal{C}_{\tilde{X}}^{\infty}\right) \\
& \rightarrow s^{-1} R j_{*} R \mathcal{H} \operatorname{Hom}_{\mathcal{D}_{\Omega}}\left(\mathcal{D}_{\Omega \rightarrow X},\left(\tilde{p}^{-1} D^{\prime} F\right) \stackrel{\mathrm{w}}{\otimes} \mathcal{C}_{\Omega}^{\infty}\right) \\
& \simeq s^{-1} R j_{*} \tilde{p}^{-1}\left(D^{\prime} F \stackrel{\mathrm{w}}{\otimes} \mathcal{C}_{X}^{\infty}\right) \\
& =\nu_{M}\left(\left(D^{\prime} F\right) \stackrel{\mathrm{w}}{\otimes} \mathcal{C}_{X}^{\infty}\right) .
\end{aligned}
$$


The second morphism follows from the fact that the Whitney functor is local and $\left.\left.\left(p^{-1} F\right)_{\bar{\Omega}}\right|_{\Omega} \simeq\left(p^{-1} F\right)\right|_{\Omega}$. The third morphism follows from the isomorphism (4), as $\tilde{p}$ is smooth.

Notice that we have a chain of morphisms:

$$
\begin{aligned}
\left(p^{-1}\left(D^{\prime} F\right)\right)_{\bar{\Omega}} & \rightarrow R \Gamma_{\Omega}\left(p^{-1} D^{\prime} F\right) \\
& \simeq R j_{*} \tilde{p}^{-1} R \mathcal{H o m}\left(F, \mathbb{C}_{X}\right) \\
& \simeq R j_{*} R \mathcal{H} \operatorname{Hom}\left(\tilde{p}^{-1} F, \mathbb{C}_{\Omega}\right) \\
& \simeq R \mathcal{H o m}\left(p^{-1} F, R \Gamma_{\Omega} \mathbb{C}_{\tilde{X}}\right) \\
& \simeq R \mathcal{H o m}\left(\left(p^{-1} F\right)_{\Omega}, \mathbb{C}_{\tilde{X}}\right) \\
& \simeq D^{\prime}\left(\left(p^{-1} F\right)_{\Omega}\right) .
\end{aligned}
$$

Then the first horizontal morphism of the proposition follows from the sequence of morphisms:

$$
\begin{aligned}
w \nu_{M}\left(D^{\prime} F, \mathcal{C}_{X}^{\infty}\right) & =s^{-1} R \mathcal{H o m} \\
& \rightarrow s^{-1} R \mathcal{H} \mathcal{D}_{\tilde{X}}\left(\mathcal{D}_{\tilde{X} \rightarrow X},\left(p^{-1} D^{\prime} F\right)_{\tilde{\Omega}} \stackrel{\mathrm{w}}{\otimes} \mathcal{C}_{\tilde{X} \rightarrow X}^{\infty}\right) \\
& \simeq T \nu_{M}\left(F, \mathcal{D} b_{X}\right)
\end{aligned}
$$

Proposition 2.3. Let $F \in \operatorname{Ob}\left(\mathbf{D}_{\mathbb{R}-c}^{\mathrm{b}}\left(\mathbb{C}_{X}\right)\right)$. In $\mathbf{D}^{\mathrm{b}}\left(\tau^{-1} \mathcal{D}_{X}\right)$, we have the isomorphism:

$$
w \nu_{M}\left(F_{M}, \mathcal{C}_{X}^{\infty}\right) \simeq \tau^{-1}\left(F_{M} \stackrel{\mathrm{w}}{\otimes} \mathcal{C}_{X}^{\infty}\right)
$$

Proof. Since $\bar{\Omega} \cap p^{-1}(M)=T_{M} X$, we have the sequence of isomorphisms:

$$
\begin{aligned}
& s^{-1} \operatorname{RHom}_{\mathcal{D}_{\tilde{X}}}\left(\mathcal{D}_{\tilde{X} \rightarrow X},\left(p^{-1} F\right)_{T_{M} X} \stackrel{\mathrm{w}}{\otimes} \mathcal{C}_{\tilde{X}}^{\infty}\right) \\
& \simeq s^{-1} R \mathcal{H o m}_{\mathcal{D}_{\tilde{X}}}\left(\mathcal{D}_{\tilde{X} \rightarrow X},\left(s_{*} s^{-1} p^{-1} F\right) \stackrel{\mathrm{w}}{\otimes} \mathcal{C}_{\tilde{X}}^{\infty}\right) \\
& \simeq s^{-1} R \mathcal{H}_{o m}{\mathcal{D}_{\tilde{X}}}\left(\mathcal{D}_{\tilde{X} \rightarrow X}, s_{*} R \mathcal{H o m}{ }_{\mathcal{D}_{T_{M} X}}\left(\mathcal{D}_{T_{M} X \rightarrow \tilde{X}},\left(s^{-1} p^{-1} F\right) \stackrel{\mathrm{w}}{\otimes} \mathcal{C}_{T_{M} X}^{\infty}\right)\right. \\
& \simeq s^{-1} s_{*} R \mathcal{H} \operatorname{Hom}_{\mathcal{D}_{T_{M} X}}\left(\mathcal{D}_{T_{M} X \rightarrow \tilde{X}} \underset{s^{-1} \mathcal{D}_{\tilde{X}}}{\stackrel{L}{\otimes}} s^{-1} \mathcal{D}_{\tilde{X} \rightarrow X},\left(s^{-1} p^{-1} F\right) \stackrel{\mathrm{w}}{\otimes} \mathcal{C}_{T_{M} X}^{\infty}\right) \\
& \simeq \text { RHom }_{\mathcal{D}_{T_{M} X}}\left(\mathcal{D}_{T_{M} X \rightarrow M} \underset{\tau^{-1} \mathcal{D}_{M}}{\stackrel{L}{\otimes}} \tau^{-1} \mathcal{D}_{M \rightarrow X},\left(\tau^{-1} i^{-1} F\right) \stackrel{\mathrm{w}}{\otimes} \mathcal{C}_{T_{M} X}^{\infty}\right) \\
& \simeq \text { RHom }_{\tau^{-1} \mathcal{D}_{M}}\left(\tau^{-1} \mathcal{D}_{M \rightarrow X}, \tau^{-1}\left(i^{-1} F \stackrel{\mathrm{w}}{\otimes} \mathcal{C}_{M}^{\infty}\right)\right) \\
& \simeq \tau^{-1} R \mathcal{H} \text { om }_{\mathcal{D}_{M}}\left(\mathcal{D}_{M \rightarrow X}, i^{-1} F \stackrel{\mathrm{w}}{\otimes} \mathcal{C}_{M}^{\infty}\right)
\end{aligned}
$$




$$
\begin{aligned}
& \simeq \tau^{-1} i^{-1} i_{*} \text { RHom }_{\mathcal{D}_{M}}\left(\mathcal{D}_{M \rightarrow X}, i^{-1} F \stackrel{\mathrm{w}}{\otimes} \mathcal{C}_{M}^{\infty}\right) \\
& \simeq \tau^{-1} i^{-1}\left(\left(i_{*} i^{-1} F\right) \stackrel{\mathrm{w}}{\otimes} \mathcal{C}_{X}^{\infty}\right) \\
& \simeq \tau^{-1} i^{-1}\left(F_{M} \stackrel{\mathrm{w}}{\otimes} \mathcal{C}_{X}^{\infty}\right) .
\end{aligned}
$$

The second and the eighth isomorphisms is a consequence of the direct image formula (2). The fifth isomorphism follows from the inverse image formula (4). The sixth isomorphism is due to the fact that $\tau$ is smooth.

Proposition 2.4 (Stalk's formula). Let $F \in \mathrm{Ob}\left(\mathbf{D}_{\mathbb{R}-c}^{\mathrm{b}}\left(\mathbb{C}_{X}\right)\right)$ and $v \in$ $T_{M} X$. Then:

$$
H^{k}\left(w \nu_{M}\left(F, \mathcal{C}_{X}^{\infty}\right)\right)_{v} \simeq \underset{U \in \mathcal{U}}{\lim _{U \in}} \mathrm{R}^{k} \Gamma\left(X ; F_{\bar{U}} \stackrel{\mathrm{w}}{\otimes} \mathcal{C}_{X}^{\infty}\right)
$$

where $\mathcal{U}$ is the family of subanalytic open subsets $U$ of $X$ such that $v \notin C_{M}(X \backslash$ $U)$.

Proof. Let $\mathcal{V}$ be a base of subanalytic relatively compact open neighborhoods of $v$ in $\tilde{X}$. We have the sequence of isomorphisms:

$$
\begin{aligned}
& H^{k}\left(w \nu_{M}\left(F, \mathcal{C}_{X}^{\infty}\right)\right)_{v} \simeq \lim _{V \in \mathcal{V}} \mathrm{R}^{k} \Gamma\left(V ; R \mathcal{H}^{\prime} m_{\mathcal{D}_{\tilde{X}}}\left(\mathcal{D}_{\tilde{X} \rightarrow X},\left(p^{-1} F\right)_{\bar{\Omega}} \stackrel{\mathrm{w}}{\otimes} \mathcal{C}_{\tilde{X}}^{\infty}\right)\right) \\
& \simeq \underset{V \in \mathcal{V}}{\lim _{V}} \mathrm{R}^{k} \Gamma\left(\tilde{X} ; R \mathcal{H}^{\prime} m_{\mathcal{D}_{\tilde{X}}}\left(\mathcal{D}_{\tilde{X} \rightarrow X}, R \Gamma_{V}\left(\left(p^{-1} F\right)_{\bar{\Omega}} \stackrel{\mathrm{w}}{\otimes} \mathcal{C}_{\tilde{X}}^{\infty}\right)\right)\right) \\
& \simeq \lim _{V \in \mathcal{V}} \mathrm{R}^{k} \Gamma\left(\tilde{X} ; R \mathcal{H}^{\prime} m_{\mathcal{D}_{\tilde{X}}}\left(\mathcal{D}_{\tilde{X} \rightarrow X},\left(p^{-1} F\right)_{\bar{\Omega} \cap \bar{V}} \stackrel{\mathrm{w}}{\otimes} \mathcal{C}_{\tilde{X}}^{\infty}\right)\right) \\
& \simeq \lim _{V \in \mathcal{V}} \mathrm{R}^{k} \Gamma\left(X ; R p_{!}\left(p^{-1} F\right)_{\bar{\Omega} \cap \bar{V}} \stackrel{\mathrm{w}}{\otimes} \mathcal{C}_{X}^{\infty}\right) \\
& \simeq \lim _{V \in \mathcal{V}} R^{k} \Gamma\left(X ; R p_{!}\left(p^{-1} F \otimes \mathbb{C}_{\bar{\Omega} \cap \bar{V}}\right) \stackrel{\mathrm{w}}{\otimes} \mathcal{C}_{X}^{\infty}\right) \\
& \simeq \underset{V \in \mathcal{V}}{\lim _{V}} \mathrm{R}^{k} \Gamma\left(X ;\left(F \otimes R p_{!} \mathbb{C}_{\bar{\Omega} \cap \bar{V}}\right) \stackrel{\mathrm{w}}{\otimes} \mathcal{C}_{X}^{\infty}\right) .
\end{aligned}
$$

The third isomorphism follows from Remark 1.4, the fourth isomorphism follows from the morphism of direct image (2) for $p$ is proper on $\operatorname{supp}\left(\left(p^{-1} F\right)_{\bar{\Omega} \cap \bar{V}}\right)$. We have the sequence of morphisms:

$$
\begin{aligned}
R p_{!} \mathbb{C}_{\bar{\Omega} \cap \bar{V}} & \simeq R p_{*} \mathbb{C}_{\bar{\Omega} \cap \bar{V}} \\
& \leftarrow R p_{*} \mathbb{C}_{p^{-1}(p(\bar{\Omega} \cap \bar{V}))} \\
& \simeq R p_{*} p^{-1} \mathbb{C}_{p(\bar{\Omega} \cap \bar{V})} \\
& \leftarrow \mathbb{C}_{p(\bar{\Omega} \cap \bar{V})}
\end{aligned}
$$


By using the same construction as in [KS2], there is an open neighborhood system $\mathcal{V}$ of $v$ satisfying the following properties:

i) $\bar{\Omega} \cap \bar{V}=\overline{\Omega \cap V}$.

ii) For $x \in X \backslash M, p^{-1}(x) \cap \bar{\Omega} \cap \bar{V}$ is either empty or isomorphic to a closed interval $[a ; b]$.

iii) For $x \in M, p^{-1}(x) \cap \bar{\Omega} \cap \bar{V}=\tau^{-1}(x) \cap \bar{V}$ is either empty or isomorphic to a compact convex set in $\tau^{-1}(x)$.

Hence we have:

$$
H^{k}\left(R p ! \mathbb{C}_{\bar{\Omega} \cap \bar{V}}\right)_{x} \simeq \begin{cases}\mathbb{C} & \text { if } k=0 \text { and } x \in p(\overline{\Omega \cap V}) \\ 0 & \text { otherwise. }\end{cases}
$$

Notice that $U=p(V \cap \Omega)$ ranges then through the family of open subanalytic subsets of $X$ such that $v \notin C_{M}(X \backslash U)$.

Proposition 2.5. $\quad$ i) If $Z$ is the inverse image of a compact subset by $\dot{\gamma}$, we can write the sections on $Z$ of the Whitney specialization for $F_{X \backslash M}$ :

$$
\mathrm{R}^{k} \Gamma\left(Z ; w \nu_{M}\left(F_{X \backslash M}, \mathcal{C}_{X}^{\infty}\right)\right) \simeq \underset{U \in \mathcal{U}}{\lim _{U}} \mathrm{R}^{k} \Gamma\left(X ; F_{\bar{U} \backslash M} \stackrel{⿱ 乛}{\otimes} \mathcal{C}_{X}^{\infty}\right)
$$

where $\mathcal{U}$ is the family of open subanalytic subsets of $X$ such that $Z \cap C_{M}(X \backslash$ $U)=\varnothing$.

ii) If moreover $\left.\tau\right|_{Z}$ has contractible stalks, we also have the same formula for the Whitney specialization of $F$ :

$$
\mathrm{R}^{k} \Gamma\left(Z ; w \nu_{M}\left(F, \mathcal{C}_{X}^{\infty}\right)\right) \simeq \underset{U \in \mathcal{U}}{\lim _{U}} \mathrm{R}^{k} \Gamma\left(X ; F_{\bar{U}} \stackrel{\mathrm{w}}{\otimes} \mathcal{C}_{X}^{\infty}\right)
$$

Proof. The proof of (12) is similar to the proof of the stalk's formula. We just have to replace the basis of neighborhoods of $v$ in $\tilde{X}$ by the family of relatively compact subanalytic open subsets of $\tilde{X}$ such that $Z \subset \mathbb{R}_{+}^{*}\left(V \cap T_{M} X\right)$.

To prove (13), we use the distinguished triangle $F_{X \backslash M} \rightarrow F \rightarrow F_{M} \stackrel{+1}{\longrightarrow}$ and the remark that if $\left.\tau\right|_{Z}$ has contractible stalks, we have the sequence of isomorphisms:

$$
\begin{aligned}
\mathrm{R}^{k} \Gamma\left(Z ; \tau^{-1}\left(F_{M} \stackrel{\mathrm{w}}{\otimes} \mathcal{C}_{X}^{\infty}\right)\right) & \simeq \mathrm{R}^{k} \Gamma\left(\tau^{-1} \tau(Z) ; \tau^{-1}\left(F_{M} \stackrel{\mathrm{w}}{\otimes} \mathcal{C}_{X}^{\infty}\right)\right) \\
& \simeq \mathrm{R}^{k} \Gamma\left(\tau(Z) ; R \tau_{*} \tau^{-1}\left(F_{M} \stackrel{\mathrm{w}}{\otimes} \mathcal{C}_{X}^{\infty}\right)\right)
\end{aligned}
$$




$$
\begin{aligned}
& \simeq \mathrm{R}^{k} \Gamma\left(\tau(Z) ; F_{M} \stackrel{\mathrm{w}}{\otimes} \mathcal{C}_{X}^{\infty}\right) . \\
& \simeq \underset{U \in \mathcal{U}}{\lim _{U}} \mathrm{R}^{k} \Gamma\left(X ; F_{\bar{U} \cap M} \stackrel{\mathrm{w}}{\otimes} \mathcal{C}_{X}^{\infty}\right) .
\end{aligned}
$$

The last isomorphism follows from the fact that the family of $\bar{U} \cap M$ when $U$ ranges through the family of subanalytic open subsets of $X$ such that $Z \cap$ $C_{M}(X \backslash U)=\varnothing$ is a neighborhood system of $\tau(Z)=\bar{Z} \cap M$.

Corollary 2.6. Let $F \in \operatorname{Ob}\left(\mathbf{D}_{\mathbb{R}-c}^{\mathrm{b}}\left(\mathbb{C}_{X}\right)\right)$. Then we have the isomorphisms:

$$
\begin{aligned}
R \tau_{*} w \nu_{M}\left(F, \mathcal{C}_{X}^{\infty}\right) & \left.\simeq\left(F \stackrel{\mathrm{w}}{\otimes} \mathcal{C}_{X}^{\infty}\right)\right|_{M}, \\
R \tau_{!} w \nu_{M}\left(F, \mathcal{C}_{X}^{\infty}\right) & \left.\simeq\left(F_{M} \stackrel{\mathrm{w}}{\otimes} \mathcal{C}_{X}^{\infty}\right)\right|_{M} \otimes \omega_{M / X},
\end{aligned}
$$

and the distinguished triangle:

$$
\left.\left.\left(F_{M} \stackrel{\mathrm{w}}{\otimes} \mathcal{C}_{X}^{\infty}\right)\right|_{M} \otimes \omega_{M / X} \rightarrow\left(F \stackrel{\mathrm{w}}{\otimes} \mathcal{C}_{X}^{\infty}\right)\right|_{M} \rightarrow R \dot{\tau}_{*}\left(\left.w \nu_{M}\left(F, \mathcal{C}_{X}^{\infty}\right)\right|_{\dot{T}_{M} X}\right) \stackrel{+1}{\longrightarrow} .
$$

Proof. The morphism (14) is defined by the sequence of morphisms:

$$
\begin{aligned}
\left.\left(F \stackrel{\mathrm{w}}{\otimes} \mathcal{C}_{X}^{\infty}\right)\right|_{M} & \simeq i^{-1}\left(F \stackrel{\mathrm{w}}{\otimes} \mathcal{C}_{X}^{\infty}\right) \\
& \simeq k^{-1} s^{-1} p^{-1}\left(F \stackrel{\mathrm{w}}{\otimes} \mathcal{C}_{X}^{\infty}\right) \\
& \rightarrow k^{-1} s^{-1} R \mathcal{H}_{o m} \mathcal{D}_{\tilde{X}}\left(\mathcal{D}_{\tilde{X} \rightarrow X},\left(p^{-1} F\right) \stackrel{\mathrm{w}}{\otimes} \mathcal{C}_{\tilde{X}}^{\infty}\right) \\
& \rightarrow k^{-1} s^{-1} R \mathcal{H}_{o m} \mathcal{D}_{\tilde{X}}\left(\mathcal{D}_{\tilde{X} \rightarrow X},\left(p^{-1} F\right)_{\bar{\Omega}} \stackrel{\mathrm{w}}{\otimes} \mathcal{C}_{\tilde{X}}^{\infty}\right)
\end{aligned}
$$

From the stalk's formula (11), this morphism is an isomorphism. Indeed if $v=(x, 0) \in T_{M} M$ then the family of open subsets $U$ such that $v \notin C_{M}(X \backslash U)$ is a neighborhood system of $x$ in $X$.

Let us construct the isomorphism (15). From the isomorphism (10), we obtain the triangle:

$$
R \tau_{!} w \nu_{M}\left(F_{X \backslash M}, \mathcal{C}_{X}^{\infty}\right) \rightarrow R \tau_{!} w \nu_{M}\left(F, \mathcal{C}_{X}^{\infty}\right) \rightarrow R \tau_{!} \tau^{-1}\left(F_{M} \stackrel{\mathrm{w}}{\otimes} \mathcal{C}_{X}^{\infty}\right) \stackrel{+1}{\longrightarrow} .
$$

Since the third term of this triangle is isomorphic to $\left.\left(F_{M} \stackrel{\mathrm{w}}{\otimes} \mathcal{C}_{X}^{\infty}\right)\right|_{M} \otimes \omega_{T_{M} X / M}$, it is sufficient to prove that the first term of the triangle is zero.

Applying Sato's triangle $R \tau_{!}(\cdot) \rightarrow R \tau_{*}(\cdot) \rightarrow R \dot{\tau}_{*}\left(\left.\cdot\right|_{\dot{T}_{M} X}\right) \stackrel{+1}{\longrightarrow}$ to $w \nu_{M}\left(F_{X \backslash M}, \mathcal{C}_{X}^{\infty}\right)$, we obtain:

$$
\begin{aligned}
R \tau_{!} w \nu_{M}\left(F_{X \backslash M}, \mathcal{C}_{X}^{\infty}\right) & \rightarrow R \tau_{*} w \nu_{M}\left(F_{X \backslash M}, \mathcal{C}_{X}^{\infty}\right) \\
& \rightarrow R \dot{\tau}_{*}\left(\left.w \nu_{M}\left(F_{X \backslash M}, \mathcal{C}_{X}^{\infty}\right)\right|_{\dot{T}_{M} X}\right) \stackrel{+1}{\longrightarrow}
\end{aligned}
$$


Let us prove that the second arrow of this triangle is an isomorphism. Let $x \in M$. Then the family $\mathcal{U}$ of open subsets $U$ of $X$ such that $C_{M}(X \backslash U) \cap$ $\dot{\tau}^{-1}(x)=\varnothing$ is the family of open neighborhoods of $x$ in $X$. Let $\mathcal{V}$ be the family of open neighborhoods of $x$ in $M$. Then we have the sequence of isomorphisms:

$$
\begin{aligned}
H^{k}\left(R \dot{\tau}_{*}\left(\left.w \nu_{M}\left(F_{X \backslash M}, \mathcal{C}_{X}^{\infty}\right)\right|_{\dot{T}_{M} X}\right)\right)_{x} & \simeq \underset{V \in \mathcal{V}}{\lim _{V \in}} \mathrm{R}^{k} \Gamma\left(V ; R \dot{\tau}_{*}\left(\left.w \nu_{M}\left(F_{X \backslash M}, \mathcal{C}_{X}^{\infty}\right)\right|_{\dot{T}_{M} X}\right)\right) \\
& \simeq{\underset{V \in \mathcal{V}}{\longrightarrow}}^{k} \Gamma\left(\dot{\tau}^{-1}(V) ; w \nu_{M}\left(F_{X \backslash M}, \mathcal{C}_{X}^{\infty}\right)\right) \\
& \simeq \mathrm{R}^{k} \Gamma\left(\dot{\tau}^{-1}(x) ; w \nu_{M}\left(F_{X \backslash M}, \mathcal{C}_{X}^{\infty}\right)\right) \\
& \simeq \varliminf_{\overrightarrow{U \in \mathcal{U}}} \mathrm{R}^{k} \Gamma\left(X ; F_{\bar{U} \backslash M} \stackrel{\mathrm{w}}{\otimes} \mathcal{C}_{X}^{\infty}\right) \\
& \simeq H^{k}\left(w^{0} \nu_{M}\left(F_{X \backslash M}, \mathcal{C}_{X}^{\infty}\right)\right)_{(x, 0)} \\
& \simeq H^{k}\left(R \tau_{*}\left(w \nu_{M}\left(F_{X \backslash M}, \mathcal{C}_{X}^{\infty}\right)\right)\right)_{(x, 0)}
\end{aligned}
$$

The third isomorphism follows from the fact that $w \nu_{M}\left(F_{X \backslash M}, \mathcal{C}_{X}^{\infty}\right)$ is conic, the fourth from formula (12) and the fifth from the stalk's formula of $w \nu_{M}\left(F_{X \backslash M}\right.$, $\left.\mathcal{C}_{X}^{\infty}\right)$

We obtain the distinguished triangle by applying Sato's triangle to $w \nu_{M}\left(F, \mathcal{C}_{X}^{\infty}\right)$ and using the two isomorphisms (14) and (15).

Remark 2.7. If $F$ is an $\mathbb{R}$-constructible sheaf (i.e. $F$ is concentrated in degree zero), the Whitney specialization of $F$ is also concentrated in degree zero. Thus the Whitney specialization induces an exact functor from the category of $\mathbb{R}$-constructible sheaves to the category of $\tau^{-1} \mathcal{D}_{X}$-modules. We shall use the same notation:

$$
w \nu_{M}\left(\cdot, \mathcal{C}_{X}^{\infty}\right): \mathbb{R}-\operatorname{cons}(X) \rightarrow \operatorname{Mod}\left(\tau^{-1} \mathcal{D}_{X}\right)
$$

We have the exact sequence of $\tau^{-1} \mathcal{D}_{X}$-modules:

$$
0 \rightarrow w \nu_{M}\left(F_{X \backslash M}, \mathcal{C}_{X}^{\infty}\right) \rightarrow w \nu_{M}\left(F, \mathcal{C}_{X}^{\infty}\right) \rightarrow \tau^{-1}\left(F_{M} \stackrel{\mathrm{w}}{\otimes} \mathcal{C}_{X}^{\infty}\right) \rightarrow 0 .
$$

Let $V$ be a conic subanalytic open subset of $T_{M} X$ such that $\left.\tau\right|_{V}$ has contractible stalks. Then we have:

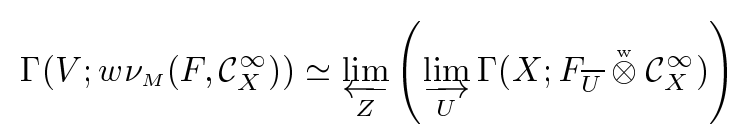

where $Z$ ranges through the family of inverse image by $\dot{\gamma}$ of subanalytic compact subsets of $\dot{\gamma}(V)$, such that $\left.\tau\right|_{Z}$ has contractible stalks, and $U$ ranges through the family of subanalytic subsets of $X$ such that $Z \cap C_{M}(X \backslash U)=\varnothing$. 


\section{$\S 2.2$. Asymptotic expansions for $C^{\infty}$-functions}

Let us consider the case where $F$ is the constant sheaf on $X$. We set:

$$
w \nu_{M}\left(\mathcal{C}_{X}^{\infty}\right)=w \nu_{M}\left(\mathbb{C}_{X}, \mathcal{C}_{X}^{\infty}\right) \quad \text { and } \quad w^{0} \nu_{M}\left(\mathcal{C}_{X}^{\infty}\right)=w \nu_{M}\left(\mathbb{C}_{X \backslash M}, \mathcal{C}_{X}^{\infty}\right) .
$$

We have a sequence of sub- $\tau^{-1} \mathcal{D}_{X}$-modules:

$$
w^{0} \nu_{M}\left(\mathcal{C}_{X}^{\infty}\right) \hookrightarrow w \nu_{M}\left(\mathcal{C}_{X}^{\infty}\right) \hookrightarrow \nu_{M}\left(\mathcal{C}_{X}^{\infty}\right) .
$$

For simplicity of exposition, we assume that $X \simeq\left\{x=(z, t) \in \mathbb{R}^{n-p} \times \mathbb{R}^{p}\right\}$ and $M \simeq\{z=0\} \simeq \mathbb{R}^{p}$. We shall call sector along $M$ a subanalytic open subset $S$ of $X$ such that $S=U_{1} \times\left(U_{2} \cap V_{2}\right)$ where $U_{1}, U_{2}$ are open convex subsets of $\mathbb{R}^{p}$ and $\mathbb{R}^{n-p}, 0 \in U_{2}$, and $V_{2}$ is an open convex cone of $\mathbb{R}^{n-p}$ with vertex at 0 . Let us denote $\tau S=U_{1} \subset M$ and $R_{+}^{*} S=U_{1} \times V_{2} \subset T_{M} X$. Let $S$ and $S^{\prime}$ be two sectors of $X$. We shall say that $S^{\prime}$ is a subsector of $S$, and write $S^{\prime}<S$, if $\bar{S}^{\prime} \backslash M \subset S$.

Definition 2.8. Let $S$ be an open sector of $X$ and $f \in \mathcal{C}^{\infty}(S)$. One says that $f$ is asymptotically developable in $S$ along $M$, if there exists a formal series $\sum_{k \in \mathbb{N}^{n-p}} a_{k}(t) z^{k}$ with $C^{\infty}$ coefficients on $\tau S$ such that

$$
\forall S^{\prime}<S, \forall m \in \mathbb{N}, \exists C>0, \forall(z, t) \in S^{\prime}, \quad\left|f(z, t)-\sum_{|k|<m} a_{k}(t) z^{k}\right| \leqslant C\|z\|^{m} .
$$

We denote by $\sigma_{M}(S)$ the vector subspace of $\mathcal{C}^{\infty}(S)$, consisting of functions asymptotically developable in $S$ along $M$. We set:

$$
\sigma_{M}^{\infty}(S)=\left\{f \in \mathcal{C}^{\infty}(S), \forall k \in \mathbb{N}^{n-p}, D^{k} f \in \sigma_{M}(S)\right\} .
$$

Proposition 2.9. Let $S$ be a sector of $X$. We have:

$$
\sigma_{M}^{\infty}(S) \simeq \lim _{S^{\prime}<S} \Gamma\left(X ; \mathbb{C}_{\bar{S}^{\prime}} \stackrel{\mathrm{w}}{\otimes} \mathcal{C}_{X}^{\infty}\right)
$$

The map which associates to a function its asymptotic expansion is a morphism of $\mathcal{D}(X)$-modules:

$$
\sigma_{M}^{\infty}(S) \stackrel{T}{\rightarrow} W_{X, \tau S}^{\infty}
$$

Proof. We have the following diagram of vector spaces:

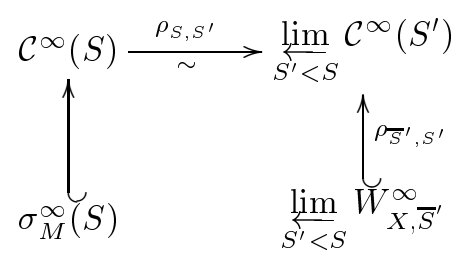


i) Let us prove that $\sigma_{M}^{\infty}(S) \subset \lim _{S^{\prime}<S} W_{X, \bar{S}^{\prime}}^{\infty}$. If $f \in \sigma_{M}^{\infty}(S)$ then for all multiindices $k \in \mathbb{N}^{n}, D^{k} f \in \sigma_{M}(S)$. From the asymptotic expansion of order 0 , we get that $D^{k} f$ is bounded on every subsector $S^{\prime}$. Since every sector is regular, it follows that $\left.D^{k} f\right|_{S^{\prime}}$ admits an extension to a continuous function on $\overline{S^{\prime}}$ and from Whitney's theorem, there exists a Whitney infinitely differentiable function $F$, on $\bar{S}^{\prime}$, such that $\left.F^{k}\right|_{S^{\prime}}=\left.D^{k} f\right|_{S^{\prime}}$.

We have the commutative diagram of $\mathcal{D}(X)$-modules:

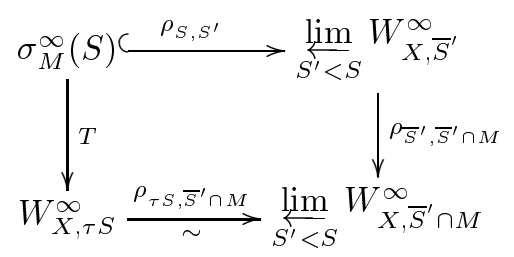

ii) Since $\lim _{S^{\prime}<S} W_{X, \bar{S}^{\prime}}^{\infty}$ is stable under derivations and $\sigma_{M}^{\infty}(S)$ is the biggest subspace of $\sigma_{M}(S)$ stable under derivations, to prove that $\varliminf_{S^{\prime}<S} W_{X, \bar{S}^{\prime}}^{\infty} \subset \sigma_{M}^{\infty}(S)$, we just have to check that $\varliminf_{S^{\prime}<S} W_{X, \bar{S}^{\prime}}^{\infty} \subset \sigma_{M}(S)$. Let $f \in \mathcal{C}^{\infty}(S)$ and $\left(F^{k}\right)_{k \in \mathbb{N} n}$ be a Whitney function on a subsector $S^{\prime}$ of $S$ such that $\forall k \in \mathbb{N}^{n},\left.F^{k}\right|_{S^{\prime}}=\left.D^{k} f\right|_{S^{\prime}}$. By definition, $\forall m \in \mathbb{N}, \quad \forall a \in \bar{S}^{\prime}, \quad \forall \varepsilon>0$, there exists a neighborhood $U_{a}$ of $a$ such that:

$$
\forall x, y \in U_{a} \cap \bar{S}^{\prime}, \quad\left|F^{0}(x)-\sum_{|k| \leqslant m} \frac{(x-y)^{k}}{k !} F^{k}(y)\right| \leqslant \varepsilon d(x, y)^{m} .
$$

Eventually taking a smaller open subset $U_{a}$, we can assume that for all $x=(z, t)$ in $\bar{S}^{\prime} \cap U_{a}$, the point $y=(0, t)$ is also in $\bar{S}^{\prime} \cap U_{a}$. Since $\bar{S}^{\prime} \cap M$ is compact, there exists a positive number $\delta$ such that $S_{\delta}^{\prime}=\left\{(z, t) \in S^{\prime} /\|z\|<\delta\right\}$ is a subset of $U=\cup_{a \in \bar{S}^{\prime} \cap M} U_{a}$.

Let $k=(i, j) \in \mathbb{N}^{n-p} \times \mathbb{N}^{p}, x=(z, t) \in S_{\delta}^{\prime}$ and $y=(0, t)$. For all $t \in \bar{S}^{\prime} \cap M$, we set $a_{i}(t)=F^{(i, 0)}(0, t) / i$ !. Then, we get:

$$
\begin{aligned}
\forall(z, t) \in S_{\delta}^{\prime}, \quad\left|f(z, t)-\sum_{|i|<m} a_{i}(t) z^{i}\right| & \leqslant \varepsilon\|z\|^{m}+\left|\sum_{|i|=m} a_{i}(t) z^{i}\right| \\
& \leqslant\|z\|^{m}\left(\varepsilon+\left|\sum_{|i|=m} a_{i}(t)\right|\right) .
\end{aligned}
$$


Notice that the formal series $\sum_{i} a_{i}(t) z^{i}$ as the image of the Whitney function $F$ by the restriction map $\rho_{\bar{S}^{\prime}, \bar{S}^{\prime} \cap M}$, is a Whitney function on $\bar{S}^{\prime} \cap M$. In particular, the coefficients $a_{i}(t)$ are $C^{\infty}$ on $\tau S^{\prime}$ and continuous on $\bar{S}^{\prime} \cap M$.

Finally, in order to satisfy the estimate in Definition 2.8, we set:

$$
\begin{aligned}
& C_{1}=\sup \left\{\left|\sum_{|i|=m} a_{i}(t)\right|, t \in \bar{S}^{\prime} \cap M\right\}, \\
& C_{2}=\sup \left\{\left|f(z, t)-\sum_{|i|<m} z^{i} a_{i}(t)\right|,(z, t) \in \overline{S^{\prime} \backslash S_{\delta}^{\prime}}\right\}, \\
& C=\max \left(\varepsilon+C_{1} ; C_{2} \delta^{-m}\right) .
\end{aligned}
$$

Proposition 2.10. Let $S$ be a sector of $X$. The sections of $w \nu_{M}\left(\mathcal{C}_{X}^{\infty}\right)$ on $\mathbb{R}_{+}^{*} S$ are the germs along $M$ of $C^{\infty}$-function asymptotically developable in $S$ as well as its derivations.

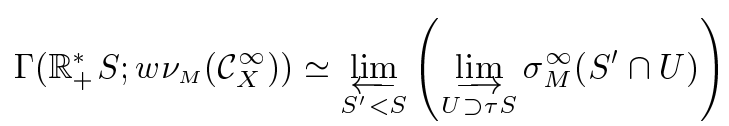

where $U$ is an open neighborhood of $\tau S$ in $X$. The space $\Gamma\left(\mathbb{R}_{+}^{*} S ; w^{0} \nu_{M}\left(\mathcal{C}_{X}^{\infty}\right)\right)$ is the subspace $\Gamma\left(\mathbb{R}_{+}^{*} S ; w \nu_{M}\left(\mathcal{C}_{X}^{\infty}\right)\right)$ of functions of asymptotic expansion zero.

The formula (21) is a consequence of the formulas (18) and (19). The second assertion follows then from (20) and the exact sequence (17):

$$
0 \rightarrow w^{0} \nu_{M}\left(\mathcal{C}_{X}^{\infty}\right) \rightarrow w \nu_{M}\left(\mathcal{C}_{X}^{\infty}\right) \stackrel{T}{\rightarrow} \tau^{-1}\left(\mathbb{C}_{M} \stackrel{⿱ 乛}{\otimes} \mathcal{C}_{X}^{\infty}\right) \rightarrow 0
$$

\section{$\S 2.3$. Operations}

Let $X$ and $Y$ be two real analytic manifolds, $M$ and $N$ two submanifolds of $X$ and $Y$, respectively. Let $p$ and $s$ be the maps associated to the normal deformation of $M \times N$ in $X \times Y$. We denote by $p^{\prime}$ and $s^{\prime}$ the maps $p_{X} \times p_{Y}$ and $s_{X} \times s_{Y}$. We have the closed embedding $k$ of $\widetilde{X \times Y}$ in $\tilde{X} \times \tilde{Y}$ which gives the commutative diagram:

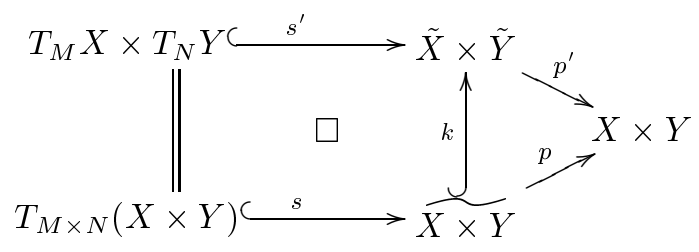


Proposition 2.11 (External tensor product).

Let $F \in \operatorname{Ob}\left(\mathbf{D}_{\mathbb{R}-c}^{\mathrm{b}}\left(\mathbb{C}_{X}\right)\right)$ and $G \in \mathrm{Ob}\left(\mathbf{D}_{\mathbb{R}-c}^{\mathrm{b}}\left(\mathbb{C}_{Y}\right)\right)$. We have the natural morphism:

$$
w \nu_{M}\left(F, \mathcal{C}_{X}^{\infty}\right) \underline{\otimes} w \nu_{N}\left(G, \mathcal{C}_{Y}^{\infty}\right) \rightarrow w \nu_{M \times N}\left((F \otimes G), \mathcal{C}_{X \times Y}^{\infty}\right)
$$

Proof. We have the sequence of morphisms:

$$
\begin{aligned}
& w \nu_{M}\left(F, \mathcal{C}_{X}^{\infty}\right) \underline{\otimes} w \nu_{N}\left(G, \mathcal{C}_{Y}^{\infty}\right) \\
& =s_{X}^{-1} R \mathcal{H o m}\left(\mathcal{D}_{\tilde{X} \rightarrow X},\left(p_{X}^{-1} F\right)_{\bar{\Omega}_{X}} \stackrel{\mathrm{w}}{\otimes} \mathcal{C}_{\tilde{X}}^{\infty}\right) \\
& \underline{\otimes} s_{Y}^{-1} \operatorname{RHom}\left(\mathcal{D}_{\tilde{Y} \rightarrow Y},\left(p_{Y}^{-1} G\right)_{\bar{\Omega}_{Y}} \stackrel{\mathrm{w}}{\otimes} \mathcal{C}_{\tilde{Y}}^{\infty}\right) \\
& \simeq s^{\prime-1} R \mathcal{H o m}\left(\mathcal{D}_{\tilde{X} \times \tilde{Y} \rightarrow X \times Y},\left(\left(p_{X}^{-1} F\right)_{\bar{\Omega}_{X}} \stackrel{\mathrm{w}}{\otimes} \mathcal{C}_{\tilde{X}}^{\infty}\right) \underline{\otimes}\left(\left(p_{Y}^{-1} G\right)_{\bar{\Omega}_{Y}} \stackrel{\mathrm{w}}{\otimes} \mathcal{C}_{\tilde{Y}}^{\infty}\right)\right) \\
& \rightarrow s^{\prime-1} R \mathcal{H o m}\left(\mathcal{D}_{\tilde{X} \times \tilde{Y} \rightarrow X \times Y},\left(\left(p_{X}^{-1} F\right)_{\bar{\Omega}_{X}} \otimes\left(p_{Y}^{-1} G\right)_{\bar{\Omega}_{Y}}\right) \stackrel{\mathrm{w}}{\otimes} \mathcal{C}_{\tilde{X} \times \tilde{Y}}^{\infty}\right) \\
& \rightarrow s^{\prime-1} R \mathcal{H o m}\left(\mathcal{D}_{\tilde{X} \times \tilde{Y} \rightarrow X \times Y},\left(R k_{!}\left(p^{-1}(F \otimes G)\right)_{\bar{\Omega}_{X \times Y}}\right) \stackrel{\mathrm{w}}{\otimes} \mathcal{C}_{\tilde{X} \times \tilde{Y}}^{\infty}\right) \\
& \simeq s^{\prime-1} R \mathcal{H o m}\left(\mathcal{D}_{\tilde{X} \times \tilde{Y} \rightarrow X \times Y}, \operatorname{Rk} k_{!} R \mathcal{H o m}\left(\mathcal{D}_{\widetilde{X \times Y} \rightarrow \tilde{X} \times \tilde{Y}},\right.\right. \\
& \left.p^{-1}(F \otimes G)_{\bar{\Omega}_{X \times Y}} \stackrel{\mathrm{w}}{\otimes} \mathcal{C} \frac{\infty}{X \times Y}\right) \\
& \simeq s^{\prime-1} R k_{!} R \mathcal{H o m}\left(\mathcal{D}_{\widetilde{X \times Y} \rightarrow \tilde{X} \times \tilde{Y}} \otimes k^{-1} \mathcal{D}_{\tilde{X} \times \tilde{Y} \rightarrow X \times Y},\right. \\
& \left.p^{-1}(F \otimes G)_{\bar{\Omega}_{X \times Y}} \stackrel{\mathrm{w}}{\otimes} \mathcal{C} \frac{\infty}{X \times Y}\right) \\
& \simeq s^{-1} R \mathcal{H o m}\left(\mathcal{D}_{\widetilde{X \times Y} \rightarrow X \times Y}, p^{-1}(F \otimes G)_{\Omega_{X \times Y}} \stackrel{\mathrm{w}}{\otimes} \mathcal{C}_{\overline{X \times Y}}^{\infty}\right) \text {. }
\end{aligned}
$$

The third morphism is a consequence of the morphism (5), the sixth morphism of the direct image formula (2) since $k$ is proper and the last morphism of the Cartesian square (23). The fourth morphism is the composition of the following morphisms:

$$
\begin{aligned}
\left(p_{X}^{-1} F\right)_{\bar{\Omega}_{X}} \otimes\left(p_{Y}^{-1} G\right)_{\bar{\Omega}_{Y}} & \simeq\left(p_{X}^{-1} F \otimes p_{Y}^{-1} G\right) \bar{\Omega}_{X} \times \bar{\Omega}_{Y} \\
& \simeq\left(p^{\prime-1}(F \otimes G) \bar{\Omega}_{X} \times \bar{\Omega}_{Y}\right. \\
& \rightarrow R k_{*} k^{-1}\left(p^{\prime-1}(F \otimes G)\right)_{\bar{\Omega}_{X} \times \bar{\Omega}_{Y}} \\
& \simeq R k_{!}\left(k^{-1} p^{\prime-1}(F \otimes G)\right)_{k^{-1}\left(\bar{\Omega}_{X} \times \bar{\Omega}_{Y}\right)} \\
& \simeq R k_{!}\left(\left(p^{-1}(F \otimes G)\right)_{\bar{\Omega}_{X \times Y}}\right) .
\end{aligned}
$$

Let $f: Y \rightarrow X$ be a morphism of real analytic manifolds, $M$ and $N$ two submanifolds respectively of $X$ and $Y$ such that $f(N) \subset M$. We have then the 
following commutative diagram where the first square is Cartesian:

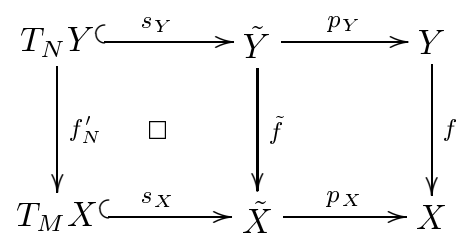

Recall that $f$ is clean relatively to $M$ if $N=f^{-1}(M)$ is a submanifold of $Y$ such that ${ }^{t} f_{N}^{\prime}: N \times_{M} T_{M}^{*} X \rightarrow T_{N}^{*} Y$ is surjective. If $N=f^{-1}(M)$ and $f$ is clean relatively to $M$, then $f_{N}^{\prime}: T_{N} Y \rightarrow N \times{ }_{M} T_{M} X$ and $p \times \tilde{f}: \tilde{Y} \rightarrow Y \times \tilde{X}$ are closed embeddings.

\section{Proposition 2.12 (Inverse image).}

Let $F \in \operatorname{Ob}\left(\mathbf{D}_{\mathbb{R}-c}^{\mathrm{b}}\left(\mathbb{C}_{X}\right)\right)$. In $\mathbf{D}^{\mathrm{b}}\left(f_{N}^{\prime}{ }^{-1} \tau^{-1} \mathcal{D}_{X}\right)$, we have the natural morphism:

$$
f_{N}^{\prime}{ }^{-1} w \nu_{M}\left(F, \mathcal{C}_{X}^{\infty}\right) \rightarrow R \mathcal{H} o_{\tau^{-1} \mathcal{D}_{Y}}\left(\tau^{-1} \mathcal{D}_{Y \rightarrow X}, w \nu_{N}\left(f^{-1} F, \mathcal{C}_{Y}^{\infty}\right)\right)
$$

If $f$ and $\left.f\right|_{N}$ are smooth, then the morphism (26) is an isomorphism. In $\mathbf{D}^{\mathrm{b}}\left(\tau^{-1} \mathcal{D}_{Y}\right)$, we have the natural morphism:

$$
\tau^{-1} \mathcal{D}_{Y \rightarrow X} \underset{\tau^{-1} f^{-1} \mathcal{D}_{X}}{\stackrel{L}{\otimes}} f_{N}^{\prime-1} w \nu_{M}\left(F, \mathcal{C}_{X}^{\infty}\right) \rightarrow w \nu_{N}\left(f^{-1} F, \mathcal{C}_{Y}^{\infty}\right) .
$$

If $f$ is a closed embedding, clean with respect to $M$ and $N=f^{-1}(M)$, then the morphism (27) is an isomorphism.

Proof. Let us first construct the morphism (26). Since $\tilde{f}^{\prime}-1\left(\bar{\Omega}_{X}\right)=\bar{\Omega}_{Y}$, we have the natural isomorphisms:

$$
\begin{aligned}
\tilde{f}^{-1}\left(p_{X}^{-1} F\right)_{\bar{\Omega}_{X}} & \simeq\left(\tilde{f}^{-1} p_{X}^{-1} F\right)_{\tilde{f}^{-1}\left(\bar{\Omega}_{X}\right)} \\
& \simeq\left(p_{Y}^{-1} f^{-1} F\right)_{\bar{\Omega}_{Y}} .
\end{aligned}
$$

Then the morphism (26) follows from the sequence of morphisms:

$$
\begin{aligned}
& {f_{N}^{\prime}}^{-1} w \nu_{M}\left(F, \mathcal{C}_{X}^{\infty}\right) \\
& \simeq s_{Y}^{-1} \tilde{f}^{-1} R \mathcal{H} \operatorname{Hom}_{\mathcal{D}_{\tilde{X}}}\left(\mathcal{D}_{\tilde{X} \rightarrow X},\left(p_{X}^{-1} F\right)_{\bar{\Omega}_{X}} \stackrel{\mathrm{w}}{\otimes} \mathcal{C}_{\tilde{X}}^{\infty}\right) \\
& \rightarrow s_{Y}^{-1} R \mathcal{H o m}_{\tilde{f}^{-1} \mathcal{D}_{\tilde{X}}}\left(\tilde{f}^{-1} \mathcal{D}_{\tilde{X} \rightarrow X}, \tilde{f}^{-1}\left(\left(p_{X}^{-1} F\right)_{\bar{\Omega}_{X}} \stackrel{\mathrm{w}}{\otimes} \mathcal{C}_{\tilde{X}}^{\infty}\right)\right) \\
& \left.\rightarrow s_{Y}^{-1} R \mathcal{H o m}_{\mathcal{D}_{\tilde{Y}}}\left(\mathcal{D}_{\tilde{Y} \rightarrow \tilde{X}} \stackrel{L}{\stackrel{L}{\otimes}} \tilde{f}^{-1} \mathcal{D}_{\tilde{X}}^{-1} \mathcal{D}_{\tilde{X} \rightarrow X},\left(\tilde{f}^{-1}\left(p_{X}^{-1} F\right)_{\bar{\Omega}_{X}}\right) \stackrel{\mathrm{w}}{\otimes} \mathcal{C}_{\tilde{Y}}^{\infty}\right)\right)
\end{aligned}
$$




$$
\begin{aligned}
& \simeq s_{Y}^{-1} \text { RHom }_{\mathcal{D}_{\tilde{Y}}}\left(\mathcal{D}_{\tilde{Y} \rightarrow Y} \stackrel{L}{\otimes} p_{p_{Y}^{-1} \mathcal{D}_{Y}}^{\otimes} p^{-1} \mathcal{D}_{Y \rightarrow X},\left(p_{Y}^{-1} f^{-1} F\right)_{\bar{\Omega}_{Y}} \stackrel{\mathrm{w}}{\otimes} \mathcal{C}_{\tilde{Y}}^{\infty}\right) \\
& \rightarrow \text { RHom }_{s_{Y}^{-1} p_{Y}^{-1} \mathcal{D}_{Y}}\left(s_{Y}^{-1} p_{Y}^{-1} \mathcal{D}_{Y \rightarrow X}, w \nu_{N}\left(f^{-1} F, \mathcal{C}_{Y}^{\infty}\right)\right)
\end{aligned}
$$

The third morphism is an application of inverse image formula (4).

If $f$ is smooth, then $\mathcal{D}_{Y \rightarrow X}$ is $\mathcal{D}_{Y}$-coherent and the last arrow is an isomorphism. If $f$ and $\left.f\right|_{N}$ are smooth, then $\tilde{f}$ is smooth and the first two arrows are also isomorphisms.

By adjunction of the morphism (26), we obtain the morphism:

$$
\tau^{-1} \mathcal{D}_{Y \rightarrow X} \underset{\tau^{-1} f^{-1} \mathcal{D}_{X}}{\stackrel{L}{\otimes}} f_{N}^{\prime-1} w \nu_{M}\left(F, \mathcal{C}_{X}^{\infty}\right) \rightarrow w \nu_{N}\left(f^{-1} F, \mathcal{C}_{Y}^{\infty}\right) .
$$

Let us prove that it is an isomorphism when $f$ is a closed embedding. Assume then that $v \in T_{N} Y$. Since $f$ and $f_{N}^{\prime}$ are closed embeddings, $\mathcal{D}_{Y \rightarrow X}$ is a right $f^{-1} \mathcal{D}_{X}$-coherent module and $R f_{N !}^{\prime} \simeq f_{N *}^{\prime}$ is exact. We have the sequence of isomorphisms:

$$
\begin{aligned}
& H^{k}\left(\tau^{-1} \mathcal{D}_{Y \rightarrow X} \underset{\tau^{-1} f^{-1} \mathcal{D}_{X}}{\stackrel{L}{\otimes}} f_{N}^{\prime}-1 w \nu_{M}\left(F, \mathcal{C}_{X}^{\infty}\right)\right)_{v} \\
& \simeq H^{k}\left(f_{N *}^{\prime} \tau^{-1} \mathcal{D}_{Y \rightarrow X} \underset{\tau^{-1} \mathcal{D}_{X}}{\stackrel{L}{\otimes}} w \nu_{M}\left(F, \mathcal{C}_{X}^{\infty}\right)\right)_{f_{N}^{\prime}(v)} \\
& \simeq \lim _{\longrightarrow} \mathrm{R}^{k} \Gamma\left(X ; f_{*} \mathcal{D}_{Y \rightarrow X} \underset{\mathcal{D}_{X}}{\stackrel{L}{\otimes}} F_{\bar{U}} \stackrel{\mathrm{w}}{\otimes} \mathcal{C}_{X}^{\infty}\right) \\
& \simeq \lim _{\longrightarrow} \mathrm{R}^{k} \Gamma\left(X ; f_{*}\left(\mathcal{D}_{Y \rightarrow X} \underset{f^{-1} \mathcal{D}_{X}}{\stackrel{L}{\otimes}} f^{-1}\left(F_{\bar{U}} \stackrel{\mathrm{w}}{\otimes} \mathcal{C}_{X}^{\infty}\right)\right)\right) \\
& \simeq \lim _{\longrightarrow} \mathrm{R}^{k} \Gamma\left(Y ;\left(f^{-1} F_{\bar{U}}\right) \stackrel{\mathrm{w}}{\otimes} \mathcal{C}_{Y}^{\infty}\right) \\
& \simeq \lim _{\longrightarrow} \mathrm{R}^{k} \Gamma\left(Y ;\left(f^{-1} F\right)_{Y \cap \bar{U}} \stackrel{\mathrm{w}}{\otimes} \mathcal{C}_{Y}^{\infty}\right) \\
& \simeq H^{k}\left(w \nu_{N}\left(f^{-1} F, \mathcal{C}_{Y}^{\infty}\right)\right)_{v} .
\end{aligned}
$$

The second and last isomorphisms follow from the stalk's formula (11). The fourth isomorphism follows from the inverse image morphism (3).

Proposition 2.13 (Direct image).

Let $G \in \operatorname{Ob}\left(\mathbf{D}_{\mathbb{R}-c}^{\mathrm{b}}\left(\mathbb{C}_{Y}\right)\right)$. In $\mathbf{D}^{\mathrm{b}}\left(\tau^{-1} \mathcal{D}_{X}\right)$, we have the natural morphism:

$$
w \nu_{M}\left(R f_{!} G, \mathcal{C}_{X}^{\infty}\right) \rightarrow R f_{N *}^{\prime} R \mathcal{H} \operatorname{Hom}_{\tau^{-1} \mathcal{D}_{Y}}\left(\tau^{-1} \mathcal{D}_{Y \rightarrow X}, w \nu_{N}\left(G, \mathcal{C}_{Y}^{\infty}\right)\right) .
$$

If $f$ is smooth, proper on $\operatorname{supp} G$, and $N=f^{-1}(M)$, then the morphism (28) is an isomorphism. 
Proof. Let us begin with the construction of the morphism (28). By adjunction in the morphism (26), we have the morphism:

$$
w \nu_{M}\left(F, \mathcal{C}_{X}^{\infty}\right) \rightarrow R f_{N *}^{\prime} R \mathcal{H} o_{\tau^{-1} \mathcal{D}_{Y}}\left(\tau^{-1} \mathcal{D}_{Y \rightarrow X}, w \nu_{N}\left(f^{-1} F, \mathcal{C}_{Y}^{\infty}\right)\right)
$$

Replacing $F$ by $R f_{!} G$, and composing with $f^{-1} R f_{!} G \rightarrow f^{-1} R f_{*} G \rightarrow G$, we obtain the morphism (28).

Let us prove that, if $f$ is smooth, proper on $\operatorname{supp} G$, and $N=f^{-1}(M)$, then the morphism (28) is an isomorphism. If $f$ is smooth and $N=f^{-1}(M)$, we assume locally on $Y$ that $f$ is the first projection of $X \times Z$ and $N=M \times Z$. Then the second square of the diagram (25) is Cartesian, and we have the sequence of isomorphisms:

$$
\begin{aligned}
\left(p_{X}^{-1} R f_{!} G\right)_{\bar{\Omega}_{X}} & \simeq\left(R \tilde{f}_{!} p_{Y}^{-1} G\right)_{\bar{\Omega}_{X}} \\
& \simeq R \tilde{f}_{!}\left(\left(p_{Y}^{-1} G\right)_{\tilde{f}^{-1}\left(\bar{\Omega}_{X}\right)}\right) \\
& \simeq R \tilde{f}_{!}\left(\left(p_{Y}^{-1} G\right)_{\bar{\Omega}_{Y}}\right)
\end{aligned}
$$

Moreover since $f$ is proper on $\operatorname{supp} G, \tilde{f}$ is proper on $\operatorname{supp}\left(p^{-1} G\right)_{\bar{\Omega}}$ and $f_{N}^{\prime}$ is proper on $\operatorname{supp}\left(p^{-1} G\right) \cap T_{M} X$. Then the isomorphism (28) follows from the sequence of isomorphisms:

$$
\begin{aligned}
& w \nu_{M}\left(R f_{!} G, \mathcal{C}_{X}^{\infty}\right) \\
& \simeq s_{X}^{-1} R \mathcal{H} \operatorname{Hom}_{\mathcal{D}_{\tilde{X}}}\left(\mathcal{D}_{\tilde{X} \rightarrow X}, R \tilde{f}_{!}\left(p_{Y}^{-1} G\right)_{\bar{\Omega}_{Y}} \stackrel{\mathrm{w}}{\otimes} \mathcal{C}_{\tilde{X}}^{\infty}\right) \\
& \simeq s_{X}^{-1} R \mathcal{H} \operatorname{Hom}_{\mathcal{D}_{\tilde{X}}}\left(\mathcal{D}_{\tilde{X} \rightarrow X}, R \tilde{f}_{*} R \mathcal{H}{ }^{\prime} m_{\mathcal{D}_{\tilde{Y}}}\left(\mathcal{D}_{\tilde{Y} \rightarrow \tilde{X}},\left(p_{Y}^{-1} G\right)_{\bar{\Omega}_{Y}} \stackrel{\mathrm{w}}{\otimes} \mathcal{C}_{\tilde{Y}}^{\infty}\right)\right) \\
& \left.\simeq s_{X}^{-1} R \tilde{f}_{*} R \mathcal{H} \operatorname{Hom}_{\mathcal{D}_{\tilde{Y}}}\left(\mathcal{D}_{\tilde{Y} \rightarrow \tilde{X}} \stackrel{L}{\stackrel{L}{\otimes}} \tilde{f}^{-1} \mathcal{D}_{\tilde{X}}^{-1} \mathcal{D}_{\tilde{X} \rightarrow X},\left(p_{Y}^{-1} G\right)_{\bar{\Omega}_{Y}} \stackrel{\mathrm{w}}{\otimes} \mathcal{C}_{\tilde{Y}}^{\infty}\right)\right) \\
& \left.\simeq R f_{N *}^{\prime} s_{Y}^{-1} R \mathcal{H} \operatorname{Hom}_{\mathcal{D}_{\tilde{Y}}}\left(\mathcal{D}_{\tilde{Y} \rightarrow Y} \stackrel{L}{\stackrel{L}{\otimes}} p_{p_{Y}^{-1} \mathcal{D}_{Y}}^{-1} \mathcal{D}_{Y \rightarrow X},\left(p_{Y}^{-1} G\right)_{\bar{\Omega}_{Y}} \stackrel{\mathrm{w}}{\otimes} \mathcal{C}_{\tilde{Y}}^{\infty}\right)\right) \\
& \simeq R f_{N *}^{\prime} s_{Y}^{-1} R \mathcal{H o m}_{p_{Y}^{-1} \mathcal{D}_{Y}}\left(p_{Y}^{-1} \mathcal{D}_{Y \rightarrow X}, R \mathcal{H} \operatorname{Hom}_{\mathcal{D}_{\tilde{Y}}}\left(\mathcal{D}_{\tilde{Y} \rightarrow Y},\left(p_{Y}^{-1} G\right)_{\bar{\Omega}_{Y}} \stackrel{\mathrm{w}}{\otimes} \mathcal{C}_{\tilde{Y}}^{\infty}\right)\right) \\
& \simeq R f_{N *}^{\prime} R \mathcal{H o m}_{s_{Y}^{-1} p_{Y}^{-1} \mathcal{D}_{Y}}\left(s_{Y}^{-1} p_{Y}^{-1} \mathcal{D}_{Y \rightarrow X}, w \nu_{N}\left(G, \mathcal{C}_{Y}^{\infty}\right)\right) \text {. }
\end{aligned}
$$

Since $f$ is smooth, $\mathcal{D}_{Y \rightarrow X}$ is $\mathcal{D}_{Y}$-coherent and the last arrow is an isomorphism.

Recall that $f$ is transversal to $M$ if $\left.{ }^{t} f^{\prime}\right|_{Y \times{ }_{X} T_{M}^{*} X}: Y \times_{X} T_{M}^{*} X \rightarrow T^{*} Y$ is injective. If $f$ is a closed embedding transversal to $M$ and $N=f^{-1}(M)$, we have the following commutative diagram where the vertical arrows are closed 
embeddings and the two squares are Cartesian.

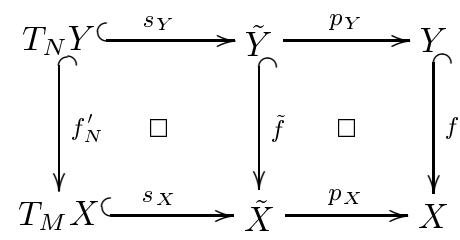

Proposition 2.14. Let $f: Y \rightarrow X$ is a closed embedding, transversal to $M$ and $N=f^{-1}(M)$. Let $g: X \rightarrow Z$ and $h: Y \rightarrow Z$ be two smooth morphisms such that $h=g \circ f$.

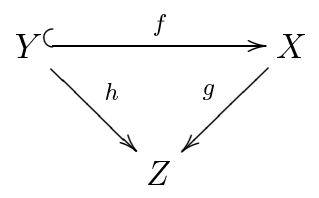

Let $G \in \operatorname{Ob}\left(\mathbf{D}_{\mathbb{R}-c}^{\mathrm{b}}\left(\mathbb{C}_{Y}\right)\right)$. We have the natural isomorphism:

$$
\operatorname{RHom}_{\mathcal{D}_{X}}\left(\mathcal{D}_{X \rightarrow Z}, w \nu_{M}\left(f_{*} G, \mathcal{C}_{X}^{\infty}\right)\right) \simeq f_{N *}^{\prime} \operatorname{RHom}_{\mathcal{D}_{Y}}\left(\mathcal{D}_{Y \rightarrow Z}, w \nu_{N}\left(G, \mathcal{C}_{Y}^{\infty}\right)\right) .
$$

Proof. Indeed from the diagram (29), we have the sequence of isomorphisms:

$$
\begin{aligned}
& R \mathcal{H o m}_{\mathcal{D}_{X}}\left(\mathcal{D}_{X \rightarrow Z}, w \nu_{M}\left(f_{*} G, \mathcal{C}_{X}^{\infty}\right)\right) \\
& =R \mathcal{H o m}_{\mathcal{D}_{X}}\left(\mathcal{D}_{X \rightarrow Z}, s_{X}^{-1} R \mathcal{H o m}_{\mathcal{D}_{\tilde{X}}}\left(\mathcal{D}_{\tilde{X} \rightarrow X},\left(p_{X}^{-1} f_{*} G\right)_{\bar{\Omega}_{X}} \stackrel{\mathrm{w}}{\otimes} \mathcal{C}_{\tilde{X}}^{\infty}\right)\right) \\
& \simeq s_{X}^{-1} R \mathcal{H o m}_{\mathcal{D}_{X}}\left(p_{X}^{-1} \mathcal{D}_{X \rightarrow Z}, R \mathcal{H o m}{ }_{\mathcal{D}_{\tilde{X}}}\left(\mathcal{D}_{\tilde{X} \rightarrow X},\left(\tilde{f}_{*} p_{Y}^{-1} G\right)_{\bar{\Omega}_{X}} \stackrel{\mathrm{w}}{\otimes} \mathcal{C}_{\tilde{X}}^{\infty}\right)\right) \\
& \simeq s_{X}^{-1} \operatorname{RHom}_{\mathcal{D}_{\tilde{X}}}\left(\mathcal{D}_{\tilde{X} \rightarrow Z},\left(\tilde{f}_{*}\left(p_{Y}^{-1} G\right)_{\bar{\Omega}_{Y}}\right) \stackrel{\mathrm{w}}{\otimes} \mathcal{C}_{\tilde{X}}^{\infty}\right) \\
& \simeq s_{X}^{-1} R \mathcal{H}^{\prime} m_{\mathcal{D}_{\tilde{X}}}\left(\mathcal{D}_{\tilde{X} \rightarrow Z}, \tilde{f}_{*} R \mathcal{H}{ } m_{\mathcal{D}_{\tilde{Y}}}\left(\mathcal{D}_{\tilde{Y} \rightarrow \tilde{X}},\left(p_{Y}^{-1} G\right)_{\bar{\Omega}_{Y}} \stackrel{\mathrm{w}}{\otimes} \mathcal{C}_{\tilde{Y}}^{\infty}\right)\right) \\
& \simeq s_{X}^{-1} \tilde{f}_{*} R \mathcal{H o m}_{\mathcal{D}_{\tilde{Y}}}\left(\mathcal{D}_{\tilde{Y} \rightarrow Z},\left(p_{Y}^{-1} G\right)_{\bar{\Omega}_{Y}} \stackrel{\mathrm{w}}{\otimes} \mathcal{C}_{\tilde{Y}}^{\infty}\right) \\
& \simeq f_{N *}^{\prime} s_{Y}^{-1} R \mathcal{H} \operatorname{Hom}_{\mathcal{D}_{Y}}\left(p_{Y}^{-1} \mathcal{D}_{Y \rightarrow Z}, R \mathcal{H o m} \mathcal{D}_{\tilde{Y}}\left(\mathcal{D}_{\tilde{Y} \rightarrow Y},\left(p_{Y}^{-1} G\right)_{\bar{\Omega}_{Y}} \stackrel{\mathrm{w}}{\otimes} \mathcal{C}_{\tilde{Y}}^{\infty}\right)\right) \\
& \simeq f_{N *}^{\prime} R \mathcal{H o m}_{\mathcal{D}_{Y}}\left(\tau^{-1} \mathcal{D}_{Y \rightarrow Z}, w \nu_{N}\left(G, \mathcal{C}_{Y}^{\infty}\right)\right) \text {. }
\end{aligned}
$$

\section{$\S 3 . \quad$ Formal Specialization}

From now on, $X$ will be a complex analytic manifold of complex dimension $d$. We denote by $\bar{X}$ the conjugate complex manifold of $X, X_{\mathbb{R}}$ the underlying 
real analytic manifold of $X$ and $M$ a real analytic submanifold of $X_{\mathbb{R}}$.

The diagonal embedding $X_{\mathbb{R}} \hookrightarrow X \times \bar{X}$ allows us to consider $X \times \bar{X}$ as a complexification of $X_{\mathbb{R}}$. If there is no fear of confusion, we often write $X$ instead of $X_{\mathbb{R}}$. For instance, we shall write $\mathcal{C}_{X}^{\infty}$ instead of $\mathcal{C}_{X_{\mathbb{R}}}^{\infty}$. We denote $\mathcal{D}_{X}$ the sheaf of rings of finite order holomorphic differential operators on $X$.

Let $F \in \operatorname{Ob}\left(\mathbf{D}_{\mathbb{R}-\mathrm{c}}^{\mathrm{b}}\left(\mathbb{C}_{X}\right)\right)$. The functor of formal cohomology is the Dolbeault complex of the Whitney functor $[\mathrm{KS} 1]$ :

$$
F \stackrel{\mathrm{w}}{\otimes} \mathcal{O}_{X}=R \mathcal{H}_{o m}{ }_{\mathcal{D}_{\bar{X}}}\left(\mathcal{O}_{\bar{X}}, F \stackrel{\mathrm{w}}{\otimes} \mathcal{C}_{X}^{\infty}\right) .
$$

In particular, if $F$ is the constant sheaf on $X$, we have $\mathbb{C}_{X} \stackrel{\mathrm{w}}{\otimes} \mathcal{O}_{X}=\mathcal{O}_{X}$. If $X=\mathbb{C}$ and $F=\mathbb{C}_{\{0\}}$, then we have:

$$
\begin{aligned}
\mathbb{C}_{\{0\}} \stackrel{\mathrm{w}}{\otimes} \mathcal{O}_{X} & \simeq R \mathcal{H o m}_{\mathcal{D}_{\bar{X}}}\left(\mathcal{O}_{\bar{X}}, \mathbb{C}_{\{0\}} \stackrel{\mathrm{w}}{\otimes} \mathcal{C}_{X}^{\infty}\right) \\
& \simeq R \mathcal{H o m} \\
& \simeq(\mathbb{C}[[z]])_{\{0\}}\left(\mathcal{O}_{\bar{X}},(\mathbb{C}[[z, \bar{z}]])_{\{0\}}\right)
\end{aligned}
$$

\section{§3.1. Specialization of the functor of formal cohomology}

Definition 3.1. Let $F \in \mathrm{Ob}\left(\mathbf{D}_{\mathbb{R}-c}^{\mathrm{b}}\left(\mathbb{C}_{X}\right)\right)$. We set:

$$
w \nu_{M}\left(F, \mathcal{O}_{X}\right)=\text { RHom }_{\tau^{-1} \mathcal{D}_{\bar{X}}}\left(\tau^{-1} \mathcal{O}_{\bar{X}}, w \nu_{M}\left(F, \mathcal{C}_{X}^{\infty}\right)\right) .
$$

We call $w \nu_{M}\left(F, \mathcal{O}_{X}\right)$ the formal specialization of $F$ along $M$.

In $\mathbf{D}^{\mathrm{b}}\left(\tau^{-1} \mathcal{D}_{X}\right)$, we have the commutative diagram:

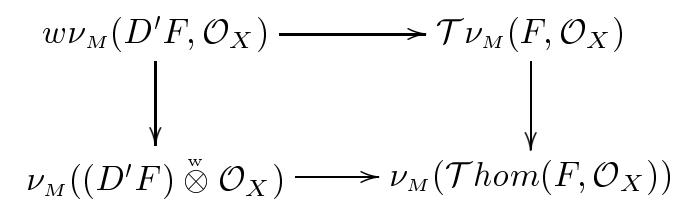

Proposition 3.2 (stalk's formula).

Let $F \in \operatorname{Ob}\left(\mathbf{D}_{\mathbb{R}-c}^{\mathrm{b}}\left(\mathbb{C}_{X}\right)\right)$ and $v \in T_{M} X$. Then:

$$
H^{k}\left(w \nu_{M}\left(F, \mathcal{O}_{X}\right)\right)_{v} \simeq \underset{U \in \mathcal{U}}{\lim _{U}} \mathrm{R}^{k} \Gamma\left(X ; F_{\bar{U}} \stackrel{\mathrm{w}}{\otimes} \mathcal{O}_{X}\right)
$$

where $\mathcal{U}$ is the family of subanalytic open subsets of $X$ such that $v \notin C_{M}(X \backslash U)$.

Proof. Let us notice first that:

$$
w \nu_{M}\left(F, \mathcal{O}_{X}\right) \simeq s^{-1} R \mathcal{H} o m_{p^{-1}} \mathcal{D}_{\bar{X}}\left(p^{-1} \mathcal{O}_{\bar{X}}, R \mathcal{H} \operatorname{Hom}_{\mathcal{D}_{\tilde{X}}}\left(\mathcal{D}_{\tilde{X} \rightarrow X},\left(p^{-1} F\right)_{\bar{\Omega}} \stackrel{\mathrm{w}}{\otimes} \mathcal{C}_{\tilde{X}}^{\infty}\right)\right) .
$$


Then we have the sequence of isomorphisms:

$$
\begin{aligned}
& H^{k}\left(w \nu_{M}\left(F, \mathcal{O}_{X}\right)\right)_{v} \\
& \simeq \lim _{\longrightarrow} \mathrm{R}^{k} \Gamma\left(V ; R \mathcal{H}^{-1} m_{p^{-1} \mathcal{D}_{\bar{X}}}\left(p^{-1} \mathcal{O}_{\bar{X}}, R \mathcal{H} \operatorname{Hom}_{\mathcal{D}_{\tilde{X}}}\left(\mathcal{D}_{\tilde{X} \rightarrow X},\left(p^{-1} F\right)_{\bar{\Omega}} \stackrel{\mathrm{w}}{\otimes} \mathcal{C}_{\tilde{X}}^{\infty}\right)\right)\right) \\
& \simeq \lim ^{k} \Gamma\left(\tilde{X} ; R^{k} \mathcal{H}^{k} m_{p^{-1}} \mathcal{D}_{\bar{X}}\left(p^{-1} \mathcal{O}_{\bar{X}}, R \mathcal{H} \operatorname{Hom}_{\mathcal{D}_{\tilde{X}}}\left(\mathcal{D}_{\tilde{X} \rightarrow X}, R \Gamma_{V}\left(\left(p^{-1} F\right)_{\bar{\Omega}} \stackrel{\mathrm{w}}{\otimes} \mathcal{C}_{\tilde{X}}^{\infty}\right)\right)\right)\right) \\
& \simeq \lim _{\longrightarrow} \mathrm{R}^{k} \Gamma\left(X ; R \mathcal{H} \operatorname{mom}_{\mathcal{D}_{\bar{X}}}\left(\mathcal{O}_{\bar{X}}, R p_{*} R \mathcal{H} \operatorname{Hom}_{\mathcal{D}_{\tilde{X}}}\left(\mathcal{D}_{\tilde{X} \rightarrow X}, R \Gamma_{V}\left(\left(p^{-1} F\right)_{\bar{\Omega}} \stackrel{\mathrm{w}}{\otimes} \mathcal{C}_{\tilde{X}}^{\infty}\right)\right)\right)\right)
\end{aligned}
$$

Following the same argument as for the computation of the stalk in the $\mathrm{C}^{\infty}$ case, we obtain:

$$
\begin{aligned}
H^{k}\left(w \nu_{M}\left(F, \mathcal{O}_{X}\right)\right)_{v} & \simeq \lim \mathrm{R}^{k} \Gamma\left(X ; R \mathcal{H o m}_{\mathcal{D}_{\bar{X}}}\left(\mathcal{O}_{\bar{X}}, F_{\bar{U}} \stackrel{\mathrm{w}}{\otimes} \mathcal{C}_{X}^{\infty}\right)\right) \\
& \simeq \varliminf_{\lim } \mathrm{R}^{k} \Gamma\left(X ; F_{\bar{U}} \stackrel{\mathrm{w}}{\otimes} \mathcal{O}_{X}\right),
\end{aligned}
$$

where $U$ ranges through the family of subanalytic open subsets of $X$ such that $v \notin C_{M}(X \backslash U)$.

Proposition 3.3. Let $F \in \mathrm{Ob}\left(\mathbf{D}_{\mathbb{R}-c}^{\mathrm{b}}\left(\mathbb{C}_{X}\right)\right)$. We have the isomorphisms:

$$
\begin{aligned}
R \tau_{*}\left(w \nu_{M}\left(F, \mathcal{O}_{X}\right)\right) & \left.\simeq\left(F \stackrel{\mathrm{w}}{\otimes} \mathcal{O}_{X}\right)\right|_{M} \\
R \tau_{!}\left(w \nu_{M}\left(F, \mathcal{O}_{X}\right)\right) & \left.\simeq\left(F_{M} \stackrel{\mathrm{w}}{\otimes} \mathcal{O}_{X}\right)\right|_{M} \otimes \omega_{M / X},
\end{aligned}
$$

and the distinguished triangle:

(36) $\left.\left.\left(F_{M} \stackrel{\mathrm{w}}{\otimes} \mathcal{O}_{X}\right)\right|_{M} \otimes \omega_{M / X} \rightarrow\left(F \stackrel{\mathrm{w}}{\otimes} \mathcal{O}_{X}\right)\right|_{M} \rightarrow R \dot{\tau}_{*}\left(\left.w \nu_{M}\left(F, \mathcal{O}_{X}\right)\right|_{\dot{T}_{M} X}\right) \stackrel{+1}{\longrightarrow}$.

Proof. To check the isomorphism (34), we consider the sequence of isomorphisms:

$$
\begin{aligned}
R \tau_{*}\left(w \nu_{M}\left(F, \mathcal{O}_{X}\right)\right) & =R \tau_{*} R \mathcal{H} \operatorname{mom}_{\tau^{-1} \mathcal{D}_{\bar{X}}}\left(\tau^{-1} \mathcal{O}_{\bar{X}}, w \nu_{M}\left(F, \mathcal{C}_{X}^{\infty}\right)\right) \\
& \simeq R \mathcal{H} m_{i^{-1} \mathcal{D}_{\bar{X}}}\left(i^{-1} \mathcal{O}_{\bar{X}}, R \tau_{*} w \nu_{M}\left(F, \mathcal{C}_{X}^{\infty}\right)\right) \\
& \simeq R \mathcal{H} m_{i^{-1} \mathcal{D}_{\bar{X}}}\left(i^{-1} \mathcal{O}_{\bar{X}}, i^{-1}\left(F \stackrel{\mathrm{w}}{\otimes} \mathcal{C}_{X}^{\infty}\right)\right) \\
& \simeq i^{-1} R \mathcal{H o m}_{\mathcal{D}_{\bar{X}}}\left(\mathcal{O}_{\bar{X}}, F_{X \backslash M} \stackrel{\mathrm{w}}{\otimes} \mathcal{C}_{X}^{\infty}\right) \\
& \left.\simeq\left(F \stackrel{\mathrm{w}}{\otimes} \mathcal{O}_{X}\right)\right|_{M} .
\end{aligned}
$$

The third isomorphism follows from the isomorphism (14) and the forth from the coherence of $\mathcal{O}_{\bar{X}}$ on $\mathcal{D}_{\bar{X}}$. 
As for the isomorphism (35), it follows from the sequence of isomorphisms:

$$
\begin{aligned}
R \tau_{!}\left(w \nu_{M}\left(F, \mathcal{O}_{X}\right)\right) & \simeq k^{!} \text {RHom }_{\tau^{-1} \mathcal{D}_{\bar{X}}}\left(\tau^{-1} \mathcal{O}_{\bar{X}}, w \nu_{M}\left(F, \mathcal{C}_{X}^{\infty}\right)\right) \\
& \simeq R \mathcal{H} \text { om }_{i^{-1} \mathcal{D}_{\bar{X}}}\left(i^{-1} \mathcal{O}_{\bar{X}}, k^{!} w \nu_{M}\left(F, \mathcal{C}_{X}^{\infty}\right)\right) \\
& \simeq R \mathcal{H} \text { om }_{i^{-1} \mathcal{D}_{\bar{X}}}\left(i^{-1} \mathcal{O}_{\bar{X}}, i^{-1}\left(F_{M} \stackrel{\mathrm{w}}{\otimes} \mathcal{C}_{X}^{\infty}\right) \otimes \omega_{M / X}\right) \\
& \simeq i^{-1} R \mathcal{H} \operatorname{Hom}_{\mathcal{D}_{\bar{X}}}\left(\mathcal{O}_{\bar{X}}, F_{M} \stackrel{\mathrm{w}}{\otimes} \mathcal{C}_{X}^{\infty}\right) \otimes \omega_{M / X} \\
& \left.\simeq\left(F_{M} \stackrel{\mathrm{w}}{\otimes} \mathcal{O}_{X}\right)\right|_{M} \otimes \omega_{M / X} .
\end{aligned}
$$

The third isomorphism follows from the isomorphism (15).

Applying Sato's triangle to $w \nu_{M}\left(F, \mathcal{O}_{X}\right)$, we obtain the distinguished triangle (36).

\section{§3.2. Complex normal deformation}

If $M$ is a complex submanifold of $X$, it will sometimes be useful to give an expression of the formal specialization in terms of the deformation to the normal complex bundle $[\mathrm{BFM}],[\mathrm{V}]$. We denote by $\tilde{X}^{c}$ the complex normal deformation, by $z$ the projection on $\mathbb{C}$ and by $\rho$ the projection on $X$. We denote by $\sigma$ the embedding of $T_{M} X$ in $\tilde{X}^{c}$. Recall that $z^{-1}(0) \simeq T_{M} X$ and $z^{-1}\left(\mathbb{C}^{*}\right) \simeq X \times \mathbb{C}^{*}$. Identifying $\tilde{X}_{\mathbb{R}}$ with $z^{-1}(\mathbb{R})$ and $\Omega$ with $z^{-1}\left(\mathbb{R}_{+}^{*}\right)$, we obtain the commutative diagram of real analytic manifolds:

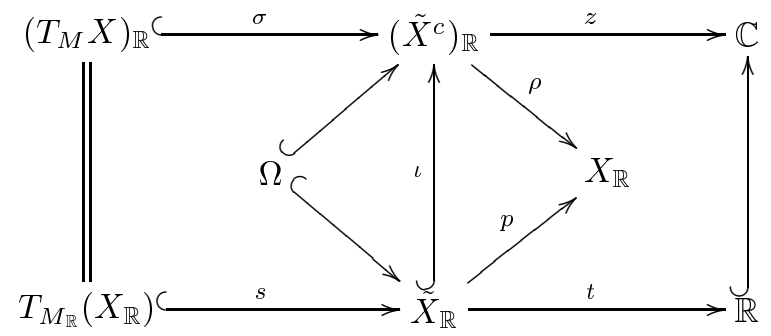

Proposition 3.4. Let $F \in \mathrm{Ob}\left(\mathbf{D}_{\mathbb{R}-c}^{\mathrm{b}}\left(\mathbb{C}_{X}\right)\right)$. We have the isomorphism:

$$
w \nu_{M}\left(F, \mathcal{O}_{X}\right) \simeq \sigma^{-1} R \mathcal{H} o m\left(\mathcal{D}_{\tilde{X}^{c} \rightarrow X},\left(\rho^{-1} F\right)_{\bar{\Omega}} \stackrel{\mathrm{w}}{\otimes} \mathcal{O}_{\tilde{X}^{c}}\right) .
$$

Proof. We have the sequence of isomorphisms:

$$
\begin{aligned}
w \nu_{M}\left(F, \mathcal{C}_{X}^{\infty}\right) & =s^{-1} R \mathcal{H o m}\left(\mathcal{D}_{\tilde{X}_{\mathbb{R}} \rightarrow X_{\mathbb{R}}},\left(p^{-1} F\right)_{\bar{\Omega}} \stackrel{\mathrm{w}}{\otimes} \mathcal{C}_{\tilde{X}}^{\infty}\right) \\
& \simeq \sigma^{-1} R \iota_{*} R \mathcal{H o m}\left(\mathcal{D}_{\tilde{X}_{\mathbb{R}} \rightarrow \tilde{X}_{\mathbb{R}}^{c}} \otimes \iota^{-1} \mathcal{D}_{\tilde{X}_{\mathbb{R}}^{c} \rightarrow X_{\mathbb{R}}},\left(\iota^{-1} \rho^{-1} F\right)_{\bar{\Omega}} \stackrel{\mathrm{w}}{\otimes} \mathcal{C}_{\tilde{X}}^{\infty}\right) \\
& \simeq \sigma^{-1} R \mathcal{H o m}\left(\mathcal{D}_{\tilde{X}_{\mathbb{R}}^{c} \rightarrow X_{\mathbb{R}}}, R \iota_{*}\left(\iota^{-1} \rho^{-1} F\right)_{\bar{\Omega}} \stackrel{\mathrm{w}}{\otimes} \mathcal{C}_{\tilde{X}^{c}}^{\infty}\right) \\
& \simeq \sigma^{-1} R \mathcal{H o m}\left(\mathcal{D}_{\tilde{X}_{\mathbb{R}}^{c} \rightarrow X_{\mathbb{R}}},\left(\rho^{-1} F\right)_{\bar{\Omega}} \stackrel{\mathrm{w}}{\otimes} \mathcal{C}_{\tilde{X}^{c}}^{\infty}\right) .
\end{aligned}
$$


On the other hand, from Lemma 5.5 of [KS1], we have the sequence of isomorphisms:

$$
\begin{aligned}
& w \nu_{M}\left(F, \mathcal{O}_{X}\right)=R \mathcal{H}{ }^{-1} \rho_{\sigma^{-1}} \mathcal{D}_{\bar{X}}\left(\sigma^{-1} \rho^{-1} \mathcal{O}_{\bar{X}}, w \nu_{M}\left(F, \mathcal{C}_{X}^{\infty}\right)\right) \\
& \simeq \sigma^{-1} R \mathcal{H}^{-1} m_{\rho^{-1}} \mathcal{D}_{\bar{X}}\left(\rho^{-1} \mathcal{O}_{\bar{X}}, \text { RHom }_{\mathcal{D}_{\tilde{X}_{\mathbb{R}}^{c}}}\left(\mathcal{D}_{\tilde{X}_{\mathbb{R}}^{c} \rightarrow X_{\mathbb{R}}},\left(\rho^{-1} F\right)_{\bar{\Omega}} \stackrel{\mathrm{w}}{\otimes} \mathcal{C}_{\tilde{X}^{c}}^{\infty}\right)\right. \\
& \simeq \sigma^{-1} R \mathcal{H} m_{\mathcal{D}_{\tilde{X}^{c}}}\left(\mathcal{D}_{\tilde{X}^{c} \rightarrow X}, R \mathcal{H o m}{\mathcal{\mathcal { D } _ { \overline { X ^ { c } } }}}\left(\mathcal{O}_{\overline{\tilde{X}^{c}}},\left(\rho^{-1} F\right)_{\bar{\Omega}} \stackrel{\mathrm{w}}{\otimes} \mathcal{C}_{\tilde{X}^{c}}^{\infty}\right)\right. \\
& \simeq \sigma^{-1} R \mathcal{H} \operatorname{Hom}_{\mathcal{D}_{\tilde{X}^{c}}}\left(\mathcal{D}_{\tilde{X}^{c} \rightarrow X},\left(\rho^{-1} F\right)_{\bar{\Omega}} \stackrel{\mathrm{w}}{\otimes} \mathcal{O}_{\tilde{X}^{c}}\right) \text {. }
\end{aligned}
$$

\section{§3.3. Examples}

In this section, we study the case where $F$ is the constant sheaf $\mathbb{C}_{X}$. We set:

$$
w \nu_{M}\left(\mathcal{O}_{X}\right)=w \nu_{M}\left(\mathbb{C}_{X}, \mathcal{O}_{X}\right) \quad \text { and } \quad w^{0} \nu_{M}\left(\mathcal{O}_{X}\right)=w \nu_{M}\left(\mathbb{C}_{X \backslash M}, \mathcal{O}_{X}\right)
$$

We have the distinguished triangle:

$$
w^{0} \nu_{M}\left(\mathcal{O}_{X}\right) \rightarrow w \nu_{M}\left(\mathcal{O}_{X}\right) \rightarrow \tau^{-1}\left(\mathbb{C}_{M} \stackrel{\mathrm{w}}{\otimes} \mathcal{O}_{X}\right) \stackrel{+1}{\longrightarrow}
$$

If $M$ is a complex submanifold or if $X$ is a complexification of $M$, then $w \nu_{M}\left(\mathcal{O}_{X}\right)$ and $\tau^{-1}\left(\mathbb{C}_{M} \stackrel{w}{\otimes} \mathcal{O}_{X}\right)$ are concentrated in degree zero. Indeed, if $v$ belongs to the normal bundle $T_{M} X$, then $H^{k}\left(w \nu_{M}\left(\mathcal{O}_{X}\right)\right)_{v} \simeq \underset{U}{\lim } \mathrm{R}^{k} \Gamma\left(X ; \mathbb{C}_{\bar{U}} \stackrel{\text { w }}{\otimes}\right.$ $\left.\mathcal{O}_{X}\right)$, where $U$ ranges through the family of subanalytic open subset of $X$ such that $v \notin C_{M}(X \backslash U)$. We can assume $U$ convex, then from A. Dufresnoy $[\mathrm{D}], \mathrm{R} \Gamma\left(X ; \mathbb{C}_{\bar{U}} \stackrel{\mathrm{w}}{\otimes} \mathcal{O}_{X}\right)$ is concentrated in degree zero, (see [DS] for another proof of the result). We have the same result for $\tau^{-1}\left(\mathbb{C}_{M} \stackrel{\mathrm{w}}{\otimes} \mathcal{O}_{X}\right)$. Moreover, the restriction morphism $\Gamma\left(X ; \mathbb{C}_{\bar{U}} \stackrel{\mathrm{w}}{\otimes} \mathcal{O}_{X}\right) \simeq \Gamma\left(X ; \mathbb{C}_{\bar{U}} \stackrel{\mathrm{w}}{\otimes} \mathcal{C}_{X}^{\infty}\right) \cap \mathcal{O}(U) \rightarrow$ $\Gamma\left(U ; \mathbb{C}_{\bar{U}} \stackrel{\mathrm{w}}{\otimes} \mathcal{O}_{X}\right) \simeq \mathcal{O}(U)$ is injective. Therefore we can identify $w \nu_{M}\left(\mathcal{O}_{X}\right)$ with the subsheaf $\mathcal{H}^{0} w \nu_{M}\left(\mathcal{O}_{X}\right)$ of the sheaf $\nu_{M}\left(\mathcal{O}_{X}\right)$, and we have an exact sequence of $\tau^{-1} \mathcal{D}_{X}$-modules:

$$
0 \rightarrow \mathcal{H}^{0} w^{0} \nu_{M}\left(\mathcal{O}_{X}\right) \rightarrow w \nu_{M}\left(\mathcal{O}_{X}\right) \rightarrow \tau^{-1}\left(\mathbb{C}_{M} \stackrel{\mathrm{w}}{\otimes} \mathcal{O}_{X}\right) \rightarrow \mathcal{H}^{1} w^{0} \nu_{M}\left(\mathcal{O}_{X}\right) \rightarrow 0
$$




\subsubsection{Complexification of a real manifold}

Proposition 3.5. $\quad$ Let $X$ be a complexification of $M$, a real analytic manifold. We have an exact sequence of $\mathcal{D}_{X}$-modules:

$$
0 \rightarrow w \nu_{M}\left(\mathcal{O}_{X}\right) \rightarrow \tau^{-1}\left(\mathcal{C}_{M}^{\infty}\right) \rightarrow \mathcal{H}^{1}\left(w^{0} \nu_{M}\left(\mathcal{O}_{X}\right)\right) \rightarrow 0,
$$

which induces on the zero-section, the exact sequence:

$$
\left.0 \rightarrow \mathcal{A}_{X}\right|_{M} \rightarrow \mathcal{C}_{M}^{\infty} \rightarrow \mathcal{H}^{1}\left(R \tau_{*}\left(w^{0} \nu_{M}\left(\mathcal{O}_{X}\right)\right)\right) \rightarrow 0 .
$$

The sheaf $w \nu_{M}\left(\mathcal{O}_{X}\right)$ can be seen as a subsheaf of $\tau^{-1}\left(\mathcal{C}_{M}^{\infty}\right)$. More precisely, let $S$ be a sector of $X$ along $M$. A section of $w \nu_{M}\left(\mathcal{O}_{X}\right)$ on the cone $\mathbb{R}_{+}^{*} S$ is the germ of an infinity differentiable function on $\tau S$ which is the boundary value along $M$ is a holomorphic function on $S$.

Proof. From the exact sequence (39):

$$
0 \rightarrow \mathcal{H}^{0} w^{0} \nu_{M}\left(\mathcal{O}_{X}\right) \rightarrow w \nu_{M}\left(\mathcal{O}_{X}\right) \rightarrow \tau^{-1} \mathcal{C}_{M}^{\infty} \rightarrow \mathcal{H}^{1} w^{0} \nu_{M}\left(\mathcal{O}_{X}\right) \rightarrow 0,
$$

we just have to prove that $\mathcal{H}^{0} w^{0} \nu_{M}\left(\mathcal{O}_{X}\right)$ is zero. In other words, we have to see that if a holomorphic function $f$ on a sector has a null asymptotic expansion, as well as all its derivatives, then this function $f$ is zero.

For simplicity, we assume $X \simeq\left\{z \in \mathbb{C}^{n}\right\} \simeq\left\{\left(z_{1}, z^{\prime}\right) \in \mathbb{C} \times \mathbb{C}^{n-1}\right\}, M \simeq$ $\{\Im z=0\}$ and consider the sector $S \simeq\left\{\left\|\Im z^{\prime}\right\|<r \Im z_{1},\|\Im z\|<r,\|\Re z\|<r\right\}$.

There exists a Whitney function $\left(F^{k}\right)_{k \in \mathbb{N}^{n}}$ on $\bar{S}$, such that $\left.F^{k}\right|_{S^{\prime}}=\left.D^{k} f\right|_{S}$ and $\left.F^{k}\right|_{M \cap \bar{S}}=0$. We set $S_{1}=S \cap\left\{z^{\prime}=0\right\} \subset \mathbb{C}$. Then $\left.F^{k}\right|_{\bar{S}_{1}}$ is holomorphic on $S_{1}$ and $\left.F^{k}\right|_{\bar{S}_{1} \cap \mathbb{R}}=0$. From Schwartz reflection principle, it implies that $\left.F^{k}\right|_{\bar{S}_{1}}=0$, which means that there is a point $\left(z_{1}, 0\right)$ in the interior of $S$ such that for all $k, D^{k} f$ vanishes at this point. Thus $f$ is identically zero.

\subsubsection{Asymptotic expansions for holomorphic functions}

In this paragraph, $M$ is a complex (smooth) submanifold of $X$. For simplicity of exposition, we can assume that $X \simeq\left\{(z, t) \in \mathbb{C}^{n-p} \times \mathbb{C}^{p}\right\}$ and $M \simeq\{z=0\}$. We shall use the same notations of a sector $S$, its projection $\tau S$, the generated cone $\mathbb{R}_{+}^{*} S$ and subsector, used in the paragraph 2.2.

We denote by $\sigma_{M}^{\text {hol }}(S)$ the set of holomorphic functions $f$ on a sector $S$ such that there exists a formal series $\sum_{k \in \mathbb{N}^{n-p}} a_{k}(t) z^{k}$ with holomorphic coefficients on $\tau S$ such that for all subsector $S^{\prime}$ of $S$, for all $m \in \mathbb{N}$, there exists a positive constant $C$ such that

$$
\forall(z, t) \in S^{\prime}, \quad\left|f(z, t)-\sum_{|k|<m} a_{k}(t) z^{k}\right| \leqslant C\|z\|^{m} .
$$


Proposition 3.6. Let $S$ be a sector of $X$. The sections of $w \nu_{M}\left(\mathcal{O}_{X}\right)$ on $\mathbb{R}_{+}^{*} S$ are the germs along $M$ of holomorphic functions asymptotically developable in $S$, having an asymptotic expansion with holomorphic coefficients.

$$
\Gamma\left(\mathbb{R}_{+}^{*} S ; w \nu_{M}\left(\mathcal{O}_{X}\right)\right) \simeq \lim _{S^{\prime}<S}\left(\underset{U \supset \tau S}{\lim _{U}} \sigma_{M}^{h o l}\left(S^{\prime} \cap U\right)\right),
$$

where $U$ is an open neighborhood of $\tau S$ in $X$.

This is a consequence of the following proposition.

Proposition 3.7. Let $S$ be a sector of $X$. We have:

$$
\sigma_{M}^{h o l}(S)=\sigma_{M}^{\infty}(S) \cap \mathcal{O}(S) \simeq \varliminf_{S^{\prime}<S} \Gamma\left(X ; \mathbb{C}_{\bar{S}^{\prime}} \stackrel{\mathrm{w}}{\otimes} \mathcal{O}_{X}\right) .
$$

Proof. First notice that for all sector $S$, we have $\Gamma\left(X ; \mathbb{C}_{\bar{S}} \stackrel{\mathrm{w}}{\otimes} \mathcal{O}_{X}\right) \simeq W_{X, \bar{S}} \cap$ $\mathcal{O}(S)$. So from the formula (19), we get:

$$
\varliminf_{S^{\prime}<S} \Gamma\left(X ; \mathbb{C}_{\bar{S}^{\prime}} \stackrel{\mathrm{w}}{\otimes} \mathcal{O}_{X}\right) \simeq \sigma_{M}^{\infty}(S) \cap \mathcal{O}(S) .
$$

Moreover by definition, $\sigma_{M}^{h o l}(S)$ and $\sigma_{M}^{\infty}(S) \cap \mathcal{O}(S)$ are subsets of $\sigma_{M}(S) \cap \mathcal{O}(S)$.

i) Let us prove the inclusion $\sigma_{M}^{\infty}(S) \cap \mathcal{O}(S) \subset \sigma_{M}^{h o l}(S)$.

Let $f \in \mathcal{O}(S)$ and $T(f)=\sum_{j, k \in \mathbb{N} n-p} a_{j, k}(t, \bar{t}) z^{j} \bar{z}^{k} \in W_{X, \tau S}^{\infty}$. For all $(\alpha, \beta) \in$ $\mathbb{N}^{n-p} \times \mathbb{N}^{p}$, we have:

$$
\begin{aligned}
D_{\bar{z}}^{\alpha} D_{\bar{t}}^{\beta} T(f) & =\sum_{j, k \in \mathbb{N}^{n}-p} \frac{(k+\alpha) !}{k !} D_{\bar{t}}^{\beta} a_{j, k+\alpha}(t, \bar{t}) z^{j} \bar{z}^{k} \\
& =T\left(D_{\bar{z}}^{\alpha} D_{\bar{t}}^{\beta} f\right) \\
& =0 .
\end{aligned}
$$

With $\beta=0$, this implies that $a_{j, k}(t, \bar{t})$ vanishes if $k \neq 0$. With $\alpha=0$, this implies that $D_{\bar{t}}^{\beta} a_{j, 0}(t, \bar{t})$ vanishes on $\tau S$, thus $a_{j, 0}(t, \bar{t})$ is holomorphic on $\tau S$.

ii) Let us prove that $\sigma_{M}^{\text {hol }}(S)$ is stable under derivation. Let us recall Cauchy's estimation: If $g$ is holomorphic on the polydisc $P(a, r)=\{x \in$ $\left.\mathbb{C}^{n} /\left|x_{i}-a_{i}\right|<r\right\}$ and continous on its closure, then for all $\alpha \in \mathbb{N}^{n}$, one has:

$$
\left|D^{\alpha} g(a)\right| \leqslant \frac{\alpha !}{r^{|\alpha|}} \sup _{P(a, r)}|g| .
$$


For all $S^{\prime}<S$ there exist a positive number $\varepsilon$ and a subsector $S_{1}<S$ such that for all $(z, t) \in S^{\prime}, P((z, t), \varepsilon\|z\|) \subset S_{1}$. For all $(z, t) \in S^{\prime}$, we have:

$$
\begin{aligned}
\left|D^{\alpha}\left(f(z, t)-\sum_{|k|<m} a_{k}(t) z^{k}\right)\right| & \leqslant \frac{\alpha !}{(\varepsilon\|z\|)^{|\alpha|}} \sup _{P(z, \varepsilon\|z\|)}\left|f(z, t)-\sum_{|k|<m} a_{k}(t) z^{k}\right| \\
& \leqslant \frac{\alpha !}{(\varepsilon\|z\|)^{|\alpha|}} C_{1}((1+\varepsilon)\|z\|)^{m} \\
& \leqslant \frac{\alpha !(1+\varepsilon)^{m}}{\varepsilon^{|\alpha|}} C_{1}\|z\|^{m-|\alpha|} \\
& \leqslant C_{2}\|z\|^{m-|\alpha|} .
\end{aligned}
$$

On the other hand, for $\alpha=(i, j) \in \mathbb{N}^{n-p} \times \mathbb{N}^{p}$, we have :

$$
D^{\alpha}\left(\sum_{|k|<m} a_{k}(t) z^{k}\right)=\sum_{|k|<m-|i|} \frac{(k+i) ! D^{j} a_{k+i}(t)}{k !} z^{k} .
$$

Finally, if we set

$$
C=C_{2}+\sup _{S^{\prime}}\left(\|z\|^{|k|-m+|\alpha|} \sum_{m-|\alpha| \leqslant|k|<m-|i|}\left|D^{j} a_{k+i}(t) \frac{(k+i) !}{k !}\right|\right),
$$

we have:

$$
\left|D^{\alpha} f(z, t)-\sum_{|k|<m-|\alpha|} \frac{(k+i) ! D^{j} a_{k+i}(t)}{k !} z^{k}\right| \leqslant\left. C\|z\|\right|^{m-|\alpha|} .
$$

Proposition 3.8. The distinguished triangle (38) induces an exact sequence of sheaves outside the zero-section:

$$
\left.\left.0 \rightarrow w^{0} \nu_{M}\left(\mathcal{O}_{X}\right)\right|_{\dot{T}_{M} X} \rightarrow w \nu_{M}\left(\mathcal{O}_{X}\right)\right|_{\dot{T}_{M} X} \stackrel{T}{\rightarrow} \dot{\tau}^{-1}\left(\mathcal{O}_{X} \hat{\mid}_{M}\right) \rightarrow 0
$$

On the zero-section we have the exact sequence:

$$
\left.0 \rightarrow \mathcal{O}_{X}\right|_{M} \rightarrow \mathcal{O}_{X} \hat{\mid}_{M} \rightarrow \mathcal{H}^{1}\left(R \tau_{*} w^{0} \nu_{M}\left(\mathcal{O}_{X}\right)\right) \rightarrow 0
$$

Proof. We have the exact sequence (39):

$$
0 \rightarrow \mathcal{H}^{0} w^{0} \nu_{M}\left(\mathcal{O}_{X}\right) \rightarrow w \nu_{M}\left(\mathcal{O}_{X}\right) \stackrel{T}{\rightarrow} \tau^{-1}\left(\mathcal{O}_{X} \hat{\mid}_{M}\right) \rightarrow \mathcal{H}^{1} w^{0} \nu_{M}\left(\mathcal{O}_{X}\right) \rightarrow 0
$$


i) If we consider the restriction to the zero section, the morphism $T$ from $\left.\left.w \nu_{M}\left(\mathcal{O}_{X}\right)\right|_{M} \simeq \mathcal{O}_{X}\right|_{M}$ to $\mathcal{O}_{X} \hat{\mid}_{M}$ is injective. Thus we get the exact sequence (45).

ii) The surjectivity of $T$ from $\left.w \nu_{M}\left(\mathcal{O}_{X}\right)\right|_{T_{M} X}$ to $\dot{\tau}^{-1}\left(\mathcal{O}_{X} \hat{\mid}_{M}\right)$ follows from the surjectivity of $T$ from $\sigma^{\text {hol }}(S)$ to $W_{X, \tau S} \cap \mathcal{O}(S)$, which is the Borel-Ritt theorem with parameters: For all formal series with holomorphic coefficients, there exists a sector $S$ and a holomorphic function on $S$ whose asymptotic expansion is exactly this formal series. There is a sketch of the proof, for instance, in Wasow's book [Wa].

In particular, if $X=\mathbb{C}$ and $M=\{0\}$, Malgrange and Sibuya had already given a construction of the sheaves $w \nu_{\{0\}}\left(\mathcal{O}_{\mathbb{C}}\right)$ and $w^{0} \nu_{\{0\}}\left(\mathcal{O}_{\mathbb{C}}\right)$ viewed on the circle $S^{1} \simeq\left(T_{M} X \backslash M\right) / \mathbb{R}_{+}^{*}$, denoted by $\mathcal{A}_{0}$ and $\mathcal{A}$, [Ml1], [Si]. The exact sequence (45) is a generalization of Malgrange's exact sequence:

$$
0 \rightarrow \mathbb{C}\{z\} \rightarrow \mathbb{C}[[z]] \rightarrow H^{1}\left(S^{1} ; \mathcal{A}_{0}\right) \rightarrow 0 .
$$

In the case of normal crossing divisor, M. A. Zurro also proved the equivalence for a holomorphic function to be strongly asymptotically developable in a polysector $S_{1} \times \ldots S_{n}$ of Majima $[\mathrm{Mj}]$, and to be a Whitney function on every closed subpolysectors [Mo], [Z]. If $M$ is a smooth complex submanifold of codimension $1, w \nu_{M}\left(\mathcal{O}_{X}\right)$ corresponds to the polysectors such that at least $n-1$ sectors $S_{i}$ are neighborhoods of 0 in $\mathbb{C}$.

\section{§3.4. Operations}

Let $X$ and $Y$ be two complex manifolds, $M$ and $N$ be two real analytic submanifolds respectively of $X_{\mathbb{R}}$ and $Y_{\mathbb{R}}$.

Proposition 3.9 (External tensor product).

Let $F \in \operatorname{Ob}\left(\mathbf{D}_{\mathbb{R}-c}^{\mathrm{b}}\left(\mathbb{C}_{X}\right)\right)$ and $G \in \operatorname{Ob}\left(\mathbf{D}_{\mathbb{R}-c}^{\mathrm{b}}\left(\mathbb{C}_{Y}\right)\right)$. We have the natural morphism:

$$
w \nu_{M}\left(F, \mathcal{O}_{X}\right) \underline{\otimes} w \nu_{N}\left(G, \mathcal{O}_{Y}\right) \rightarrow w \nu_{M \times N}\left((F \otimes G), \mathcal{O}_{X \times Y}\right)
$$

Proof. We just have to apply the functor $R \mathcal{H}^{\circ} m_{\mathcal{D}_{\bar{X} \times \bar{Y}}}\left(\mathcal{O}_{\bar{X} \times \bar{Y}}, \cdot\right)$ to the morphism (24):

$$
w \nu_{M}\left(F, \mathcal{O}_{X}\right) \underline{\Xi} w \nu_{N}\left(G, \mathcal{O}_{Y}\right)
$$




$$
\begin{aligned}
& \simeq \operatorname{RHom}_{\mathcal{D}_{\bar{X}}}\left(\mathcal{O}_{\bar{X}}, w \nu_{M}\left(F, \mathcal{C}_{X}^{\infty}\right)\right) \underline{\otimes} \operatorname{RHom}_{\mathcal{D}_{\bar{Y}}}\left(\mathcal{O}_{\bar{Y}}, w \nu_{N}\left(G, \mathcal{C}_{Y}^{\infty}\right)\right) \\
& \simeq R \mathcal{H o m}_{\mathcal{D}_{\bar{X} \times \bar{Y}}}\left(\mathcal{O}_{\bar{X} \times \bar{Y}}, w \nu_{M}\left(F, \mathcal{C}_{X}^{\infty}\right) \underline{\otimes} w \nu_{N}\left(G, \mathcal{C}_{Y}^{\infty}\right)\right) \\
& \rightarrow R \mathcal{H o m}_{\mathcal{D}_{\bar{X} \times \bar{Y}}}\left(\mathcal{O}_{\bar{X} \times \bar{Y}}, w \nu_{M \times N}\left((F \otimes G), \mathcal{C}_{X \times Y}^{\infty}\right)\right) \\
& \simeq w \nu_{M \times N}\left((F \otimes G), \mathcal{O}_{X \times Y}\right) .
\end{aligned}
$$

Let $f: Y \rightarrow X$ be a morphism of complex analytic manifolds, $M$ and $N$ be two real analytic submanifolds respectively of $X_{\mathbb{R}}$ and $Y_{\mathbb{R}}$.

Proposition 3.10 (Inverse image).

Let $F \in \operatorname{Ob}\left(\mathbf{D}_{\mathbb{R}-c}^{\mathrm{b}}\left(\mathbb{C}_{X}\right)\right)$. In $\mathbf{D}^{\mathrm{b}}\left(f_{N}^{\prime}{ }^{-1} \tau^{-1} \mathcal{D}_{X}\right)$, we have the natural morphism:

$$
{f_{N}^{\prime}}^{-1} w \nu_{M}\left(F, \mathcal{O}_{X}\right) \rightarrow R \mathcal{H o m}{\tau^{-1} \mathcal{D}_{Y}}\left(\tau^{-1} \mathcal{D}_{Y \rightarrow X}, w \nu_{N}\left(f^{-1} F, \mathcal{O}_{Y}\right)\right)
$$

If $f$ and $\left.f\right|_{N}$ are smooth, then the morphism (47) is an isomorphism. In $\mathbf{D}^{\mathrm{b}}\left(\tau^{-1} \mathcal{D}_{Y}\right)$, we have the natural morphism:

$$
\tau^{-1} \mathcal{D}_{Y \rightarrow X} \stackrel{L}{\otimes}{ }_{\tau^{-1} f^{-1} \mathcal{D}_{X}} f^{\prime-1} w \nu_{M}\left(F, \mathcal{O}_{X}\right) \rightarrow w \nu_{N}\left(f^{-1} F, \mathcal{O}_{Y}\right) .
$$

If $f$ is a closed embedding, clean with respect to $M$ and $N=f^{-1}(M)$, then the morphism (48) is an isomorphism.

Proof. The morphism (47) follows from the sequence of morphisms:

$$
\begin{aligned}
& f_{N}^{\prime}{ }^{-1} w \nu_{M}\left(F, \mathcal{O}_{X}\right)=f_{N}^{\prime}{ }^{-1} R \mathcal{H o m}{ }_{\tau^{-1} \mathcal{D}_{\bar{X}}}\left(\tau^{-1} \mathcal{O}_{\bar{X}}, w \nu_{M}\left(F, \mathcal{C}_{X}^{\infty}\right)\right) \\
& \simeq R \mathcal{H o m}{ }_{f_{N}^{\prime}{ }^{-1} \tau^{-1} \mathcal{D}_{\bar{X}}}\left(f_{N}^{\prime}{ }^{-1} \tau^{-1} \mathcal{O}_{\bar{X}}, f_{N}^{\prime}{ }^{-1} w \nu_{M}\left(F, \mathcal{C}_{X}^{\infty}\right)\right) \\
& \rightarrow R \mathcal{H o m}{\tau^{-1} f^{-1} \mathcal{D}_{\bar{X}}}\left(\tau^{-1} f^{-1} \mathcal{O}_{\bar{X}}, R \mathcal{H o m}\left(\tau^{-1} \mathcal{D}_{Y_{\mathbb{R}} \rightarrow X_{\mathbb{R}}}, w \nu_{N}\left(f^{-1} F, \mathcal{C}_{Y}^{\infty}\right)\right) .\right.
\end{aligned}
$$

The second morphism is an application of the direct image formula (26). If moreover $f$ and $\left.f\right|_{N}$ are smooth, then both arrows are isomorphisms.

As in Lemma 5.5 of [KS1], since $\tau^{-1} \mathcal{D}_{Y_{\mathbb{R}} \rightarrow X_{\mathbb{R}}} \simeq\left(\tau^{-1} \mathcal{D}_{Y_{\mathbb{R}}} \stackrel{{ }^{L}}{\stackrel{\sim}{\otimes}} \tau_{\tau^{-1}} \tau^{-1} \mathcal{D}_{Y \rightarrow X}\right)$ $\stackrel{L}{\otimes} \tau_{\tau^{-1} \mathcal{D}_{\bar{Y}}}^{-1} \tau^{-1} \mathcal{D}_{\bar{Y} \rightarrow \bar{X}}$, we have the isomorphisms:

$$
\begin{aligned}
& R \mathcal{H o m} \tau_{\tau^{-1} f^{-1} \mathcal{D}_{\bar{X}}}\left(\tau^{-1} f^{-1} \mathcal{O}_{\bar{X}}, R \mathcal{H o m} \tau_{\tau^{-1} \mathcal{D}_{Y_{\mathbb{R}}}}\left(\tau^{-1} \mathcal{D}_{Y_{\mathbb{R}} \rightarrow X_{\mathbb{R}}}, w \nu_{N}\left(f^{-1} F, \mathcal{C}_{Y}^{\infty}\right)\right)\right) \\
& \simeq R \mathcal{H}^{\circ} m_{\tau^{-1} \mathcal{D}_{Y}}\left(\tau^{-1} \mathcal{D}_{Y \rightarrow X}, R \mathcal{H} \operatorname{Hom}_{\tau^{-1} D_{\bar{Y}}}\left(\tau^{-1} \mathcal{O}_{\bar{Y}}, w \nu_{N}\left(f^{-1} F, \mathcal{C}_{Y}^{\infty}\right)\right)\right) \\
& \simeq R \mathcal{H o m} \tau_{\tau^{-1} \mathcal{D}_{Y}}\left(\tau^{-1} \mathcal{D}_{Y \rightarrow X}, w \nu_{N}\left(f^{-1} F, \mathcal{O}_{Y}\right)\right) \text {. }
\end{aligned}
$$


By adjunction of the morphism (47), we obtain the morphism (48). Then to prove that this morphism is an isomorphism if $f$ is a closed embedding clean relatively to $M$ and $N=f^{-1}(M)$, we have to compute the stalk exactly like in the proof of the isomorphism 27 , replacing $\cdot{ }^{\mathrm{w}} \mathcal{C}_{X}^{\infty}$ by $\cdot{ }^{\mathrm{w}} \mathcal{O}_{X}$, and using the stalk formula (33) instead the stalk formula (11).

Proposition 3.11 (Direct image).

Let $G \in \operatorname{Ob}\left(\mathbf{D}_{\mathbb{R}-c}^{\mathrm{b}}\left(\mathbb{C}_{Y}\right)\right)$. In $\mathbf{D}^{\mathrm{b}}\left(\tau^{-1} \mathcal{D}_{X}\right)$, we have the natural morphism:

$$
w \nu_{M}\left(R f_{!} G, \mathcal{O}_{X}\right) \rightarrow R f_{N *}^{\prime} R \mathcal{H} o_{\tau^{-1} \mathcal{D}_{Y}}\left(\tau^{-1} \mathcal{D}_{Y \rightarrow X}, w \nu_{N}\left(G, \mathcal{O}_{Y}\right)\right)
$$

If $f$ is smooth, proper on $\operatorname{supp} G$, and $N=f^{-1}(M)$, then the morphism (49) is an isomorphism.

Proof. We consider the sequence of morphisms:

$$
\begin{aligned}
& w \nu_{M}\left(R f_{!} G, \mathcal{O}_{X}\right)=R \mathcal{H o m}_{\tau^{-1} \mathcal{D}_{\bar{X}}}\left(\tau^{-1} \mathcal{O}_{\bar{X}}, w \nu_{M}\left(R f_{!} G, \mathcal{C}_{X}^{\infty}\right)\right)
\end{aligned}
$$

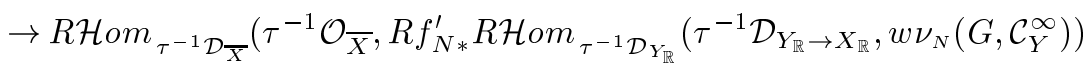

$$
\begin{aligned}
& \simeq R f_{N *}^{\prime} R \mathcal{H o m}_{f_{N}^{\prime}{ }^{-1} \tau^{-1} \mathcal{D}_{\bar{X}}} \\
& \left(f_{N}^{\prime}{ }^{-1} \tau^{-1} \mathcal{O}_{\bar{X}}, R \mathcal{H} \text { or }_{\tau^{-1}} \mathcal{D}_{Y_{\mathbb{R}}}\left(\tau^{-1} \mathcal{D}_{Y_{\mathbb{R}} \rightarrow X_{\mathbb{R}}}, w \nu_{N}\left(G, \mathcal{C}_{Y}^{\infty}\right)\right)\right) \\
& \simeq R f_{N *}^{\prime} R \mathcal{H}_{\text {om }}{ }_{\tau^{-1} f^{-1}} \mathcal{D}_{\bar{X}} \\
& \left(\tau^{-1} f^{-1} \mathcal{O}_{\bar{X}}, \text { RHom }_{\tau^{-1} \mathcal{D}_{Y_{\mathbb{R}}}}\left(\tau^{-1} \mathcal{D}_{Y_{\mathbb{R}} \rightarrow X_{\mathbb{R}}}, w \nu_{N}\left(G, \mathcal{C}_{Y}^{\infty}\right)\right)\right) \\
& \simeq R f_{N *}^{\prime} R \mathcal{H o m}_{\tau^{-1} \mathcal{D}_{Y}}\left(\tau^{-1} \mathcal{D}_{Y \rightarrow X}, w \nu_{N}\left(G, \mathcal{O}_{Y}\right)\right) \text {. }
\end{aligned}
$$

If moreover $f$ is smooth and $N=f^{-1}(M)$, then the first arrow is an isomorphism.

The following corollary is the specialization's version of morphism (5.19) of $[\mathrm{KS} 1]$ in the smooth case.

Corollary 3.12. Let $F \in \mathrm{Ob}\left(\mathbf{D}_{\mathbb{R}-c}^{\mathrm{b}}\left(\mathbb{C}_{X}\right)\right)$. If $f$ and $\left.f\right|_{N}$ are smooth, we have the natural morphism in $\mathbf{D}^{\mathrm{b}}\left(\tau^{-1} \mathcal{D}_{X}\right)$ :

$$
R f_{N !}^{\prime}\left(\tau^{-1} \mathcal{D}_{X \leftarrow Y} \stackrel{L}{\mathcal{D}_{Y}} w \nu_{N}\left(f^{-1} F, \mathcal{O}_{Y}\right)\right)\left[d_{Y}\right] \rightarrow w \nu_{M}\left(F, \mathcal{O}_{X}\right)\left[d_{X}\right]
$$


Proof. We have the sequence of morphisms:

$$
\begin{aligned}
& R f_{N !}^{\prime}\left(\tau^{-1} \mathcal{D}_{X \leftarrow Y} \stackrel{L}{\otimes} w \nu_{N}\left(f^{-1} F, \mathcal{O}_{Y}\right)\right)\left[d_{Y}\right] \\
& \simeq R f_{N !}^{\prime} R \mathcal{H}^{\prime} m_{\mathcal{D}_{Y}}\left(\tau^{-1} \mathcal{D}_{Y \rightarrow X}, w \nu_{N}\left(f^{-1} F, \mathcal{O}_{Y}\right)\right)\left[2 d_{Y}-d_{X}\right] \\
& \simeq R f_{N !}^{\prime} f_{N}^{\prime-1} w \nu_{M}\left(F, \mathcal{O}_{X}\right)\left[2 d_{Y}-d_{X}\right] \\
& \simeq R f_{N !}^{\prime} f_{N}^{\prime} ! w \nu_{M}\left(F, \mathcal{O}_{X}\right)\left[d_{X}\right] \\
& \rightarrow w \nu_{M}\left(F, \mathcal{O}_{X}\right)\left[d_{X}\right] \text {. }
\end{aligned}
$$

Proposition 3.13. Let $f: Y \rightarrow X$ be a closed embedding, transversal to $M$ and $N=f^{-1}(M)$. Let $g: X \rightarrow Z$ and $h: Y \rightarrow Z$ be two smooth morphisms such that $h=g \circ f$. Let $G \in \mathrm{Ob}\left(\mathbf{D}_{\mathbb{R}-c}^{\mathrm{b}}\left(\mathbb{C}_{Y}\right)\right)$. We have the natural isomorphism:

$$
R \mathcal{H o m} \overline{\mathcal{D}}_{X}\left(\mathcal{D}_{X \rightarrow Z}, w \nu_{M}\left(f_{*} G, \mathcal{O}_{X}\right)\right) \simeq f_{N *}^{\prime} R \mathcal{H} o_{\mathcal{D}_{Y}}\left(\mathcal{D}_{Y \rightarrow Z}, w \nu_{N}\left(G, \mathcal{O}_{Y}\right)\right) .
$$

Proof. We have the sequence of isomorphisms:

$$
\begin{aligned}
& R \mathcal{H o m}_{\mathcal{D}_{X}}\left(\mathcal{D}_{X \rightarrow Z}, w \nu_{M}\left(f_{*} G, \mathcal{O}_{X}\right)\right) \\
& \simeq \operatorname{RHom}_{\mathcal{D}_{X}}\left(\mathcal{D}_{X \rightarrow Z}, R \mathcal{H o m}{ }_{D_{\bar{X}}}\left(\mathcal{O}_{\bar{X}}, w \nu_{M}\left(f_{*} G, \mathcal{C}_{X}^{\infty}\right)\right)\right) \\
& \simeq \text { RHom }_{h^{-1} D_{\bar{X}}}\left(h^{-1} \mathcal{O}_{\bar{X}}, \text { RHom }_{\mathcal{D} X_{\mathbb{R}}}\left(\mathcal{D}_{X_{\mathbb{R}} \rightarrow Z_{\mathbb{R}}}, w \nu_{M}\left(f_{*} G, \mathcal{C}_{X}^{\infty}\right)\right)\right) \\
& \simeq \text { RHom }_{h^{-1} D_{\bar{X}}}\left(h^{-1} \mathcal{O}_{\bar{X}}, f_{*} R \mathcal{H}^{\prime} \operatorname{mom}_{\mathcal{D}_{Y_{\mathbb{R}}}}\left(\mathcal{D}_{Y_{\mathbb{R}} \rightarrow Z_{\mathbb{R}}}, w \nu_{N}\left(G, \mathcal{C}_{Y}^{\infty}\right)\right)\right) \\
& \simeq f_{*} R \mathcal{H o m}_{g^{-1} D_{\bar{X}}}\left(g^{-1} \mathcal{O}_{\bar{X}}, R \mathcal{H o m}{ }_{\mathcal{D}_{Y_{\mathbb{R}}}}\left(\mathcal{D}_{Y_{\mathbb{R}} \rightarrow Z_{\mathbb{R}}}, w \nu_{N}\left(G, \mathcal{C}_{Y}^{\infty}\right)\right)\right) \\
& \simeq f_{*} \text { RHom }_{\mathcal{D}_{Y}}\left(\mathcal{D}_{Y \rightarrow Z}, R \mathcal{H o m}{ }_{D_{\bar{X}}}\left(\mathcal{O}_{\bar{X}}, w \nu_{N}\left(G, \mathcal{C}_{Y}^{\infty}\right)\right)\right) \\
& \simeq f_{*} R \mathcal{H o m}_{\mathcal{D}_{Y}}\left(\mathcal{D}_{Y \rightarrow Z}, w \nu_{N}\left(G, \mathcal{O}_{Y}\right)\right) \text {. }
\end{aligned}
$$

\section{References}

[A] Andronikof, E., Microlocalisation tempérée, Mém. Soc. Math. Fr., 57 (1994), Suppl. Bull. 122.

[BFM] Baum, P., Fulton, W. and Macpherson, R., Riemann-Roch for singular varieties, Publ. Math. del'I. H. E. S., 45 no. 2 (1975), 101-146.

[C1] Colin, V., Spécialisation du foncteur de Whitney, C. R. Acad. Sci. Paris Sér. I Math., 323 (1996), 383-388.

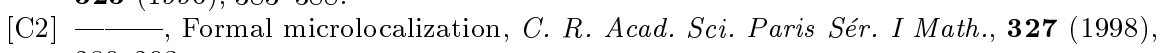
$289-293$. 
[DS] D'Agnolo, A. and Schapira, P., Leray's quantization of projective duality, Duke Math. J., 84 no. 2 (1996), 453-495.

Quantification de Leray de la dualité projective, C. R. Acad. Sci. Paris Sér. I Math., 319 (1994), 595-598.

[D] Dufresnoy, A., Sur l'opérateur d" et les fonctions différentiables au sens de Whitney, Ann. Inst. Fourier, Grenoble, 291 (1979), 229-238.

[K] Kashiwara, M., The Riemann-Hilbert problem for holonomic systems, Publ. RIMS, Kyoto Univ., 20 (1984), 319-365.

[KS1] Kashiwara, M. and Schapira, P., Moderate and formal cohomology associated with constructible sheaves, Mém. Soc. Math. France, 64, Suppl. Bull. S.M.F. 124 fasc 1 (1996).

Moderate and formal cohomology associated with constructible sheaves, Prépub. RIMS, 999 (1994).

[KS2] —— Sheaves on Manifolds, Grundlehren Math. Wiss., 292, Springer-Verlag (1990).

[KS3] - Microlocal study of sheaves, Astérisque, 128 (1985).

[Ł] Łojasiewicz, S., Sur le problème de la division, Studia Mathematica, XVIII (1959), $87-136$.

[Mj] Majima, H., Asymptotic analysis for integrable connections with irregular singular points, Lecture Notes in Math., 1075, Springer-Verlag (1984).

[M11] Malgrange, B., Remarques sur les équations différentielles à points singuliers, Lecture Notes in Math., 712, Springer-Verlag (1979).

[M12] —_ Ideals of differentiable functions, Oxford University Press, 1966.

[Mo] Mozo, J., Teoremas de división y de Malgrange-Sibuya para funciones con desarollo asintótico fuerte en varias variables, Tesis de doctorado, Universidad de Valladolid (1996).

[Sa] Sato, M., Hyperfonctions and partial differential equations, Proc. Int. Conf., on Functional Analysis and related topics, Tokyo Univ. Press (1969), 91-94.

[SKK] Sato, M., Kawai, T. and Kashiwara, M., Hyperfonctions and pseudo-differential equations, Lecture Notes in Math., 287, Springer-Verlag (1973), 265-529.

[Si] Sibuya, Y., Linear ordinary differential equations in the complex domain: Problems of analytic continuation, Progr. Math. Trans. Math. Monogr., 82, AMS (1990).

[V] Verdier, J.-L., Le théorème de Riemann-Roch pour les intersections complètes, dans Séminaire de géométrie algébrique, Astérisque, 36-37 (1976), 189-228.

[Wa] Wasow, W. R., Asymptotic expansions of ordinary differential equations, Interscience Publ, New York, 1965.

[Wh1] Whitney, H., Analytic expansion of differentiable functions defined in closed sets, Trans. of AMS, 36 (1934), 63-89.

[Wh2] —_, Functions differentiable in the boundary of regions, Ann. of Math., 35 no. 3 (1934), 482-485.

[Z] Zurro, M. A., A new Taylor type formula and $\mathcal{C}^{\infty}$ extensions for asymptotically developable functions, Studia Math., 123 no. 2 (1997), 151-163. 